\title{
4.2 Numerical and experimental study of preparation processes of liquid grain feed
}

\begin{abstract}
Numerical and experimental study of heat and mass transfer processes in rotarypulsation apparatus for the preparation of liquid grain feed has been carried out. The basic hydraulic and thermal characteristics of such devices are received and geometrical characteristics of such device are defined. An experimental sample of a device with rectangular holes in rotor-stator system was developed and manufactured. Experimental studies of such characteristics as granulometric composition of grain feed mixture, power consumption in feed production and temperature change of water-grain mixture during its processing have been carried out.
\end{abstract}

\section{Introduction}

Existing devices for the production of feed for livestock in the vast majority are based on the use of technologies for the preparation of solid feed mixtures. The basis of such technologies is the use of hammer rotors, the rotation of which is the grinding of grain or other solid components of the feed. At contact of a surface of a hammer with grain owing to shock loadings there is its cracking and formation of the mix having the different size of fractions. The design of the hammer rotors can be different, for example, in the form of cylindrical rollers or disks. The size and geometry of the surface of the hammers are selected depending on the size of the fractions of the feed mixture to be obtained at the outlet of the hammer crusher. Crusher designs with airflow can be used to improve the operation of the crushers. The main purpose in the process of preparation is to obtain a feed mixture in which the size of the feed particles are optimal for assimilation during feeding of cattle with minimal energy costs per unit of feed mass.

In [233-240], studies were conducted to develop new designs of hammer crushers, which have advantages in terms of optimal grain grinding, reduction of energy consumption per unit of output and reducing the share of dust fractions in the 
feed mixture. In the monograph [233] various types of crushers, their designs are considered, theoretical and experimental data on preparation of firm forage mixes are resulted. In [234], a two-stage crusher was developed, which improves the throughput and particle size distribution of the produced feed. The influence of air flows on improving the efficiency of hammer crushers was studied in [235, 236]. Of great importance in the operation of crushers is the amount of specific energy consumption spent on the preparation of feed. These issues were studied in the authors of [237, 238], where the optimal designs and modes of operation of two-stage and three-stage roller crushers are proposed. The amount of energy consumption required to prepare a unit mass of feed products when grinding different types of products in a hammer crusher was studied in [239]. The study of the specific energy consumption and fractional composition of grain feed in the disk crusher is devoted to [240].

Known designs of hammer, roller and disc crushers, which are the most common devices for preparing feed, can have a number of disadvantages: large volume and weight; significant specific energy consumption for the preparation of feed products; unbalanced granular composition of feed products (the presence of small fractions that are poorly digested by cattle); dry feed is inferior to liquid feed in its nutritional properties and digestibility for pigs, young cattle, sheep, goats and other animals. In this regard, it is of interest to develop new designs of devices for the preparation of liquid feed mixtures, the principle of which is based on the use of other technologies with lower energy consumption to produce a unit mass of feed product. From this point of view, technologies in which rotary-pulsation mechanisms of product processing are used are promising. Such technologies are used in the processes of preparation of food, pastes, medicines and other products for various purposes.

The main designs of rotary-pulsation devices and the processes that take place in them are considered in the monograph [241]. In [242], hydrodynamics and heat exchange in rotating-pulsating flows, which take place in rotary-pulsating devices, were studied. A number of works are devoted to the use of rotor-pulsation technologies in specific technological processes. In [243] such technologies were used in the processes of grinding and dissolving polydisperse materials, which were studied 
experimentally and by numerical simulation methods. In [244] a new design of a rotorpulsation apparatus with holes of a special shape was proposed and the formation of various emulsions in this apparatus was investigated. The study of the mechanisms that occur in the rotor-pulsation apparatus in the processing of heterogeneous materials was carried out in [245]. In [246] rotor-pulsation principles were used for preparation of liquid feed mixtures on a grain basis. In [246] the results of researches of hydraulic characteristics of the rotor-pulsation device at processing of a grain mix with water are resulted. The processes that take place in rotary-pulsation apparatus for the preparation of liquid grain feed are insufficiently studied, and the design of such devices requires further improvement.

\subsubsection{Numerical modeling of rotor-pulsation apparatus with rectangular holes (3D model).}

The following section presents the results of numerical simulations in the working chamber of RPA with rectangular holes using a 3D model. In Fig. 1 schematically shows a rotary pulsation apparatus (RPA) for feed preparation. The boot device is made in SOLID WORKS, then loaded into ANSYS Design Modeler. The Fill component is selected to simulate the mixture. Boundary conditions were set and the project was transferred to ANSYS Meshing.

Fig. 2 shows the boundary conditions of RPA. At the inlet limit of the inlet, a mixture with a mass flow rate of $0.92 \mathrm{~kg} / \mathrm{s}$ with a temperature of $+20^{\circ} \mathrm{C}$ is loaded. The output limit of the outlet is set by pressure (pressure outlet). RPA case is made of aluminum. The temperature $+20{ }^{\circ} \mathrm{C}$ is set on the surface of the metal case. The volume of RPA stator is $2.181 \cdot 10^{-3} \mathrm{~m}^{3}$ (Fig. 1). 


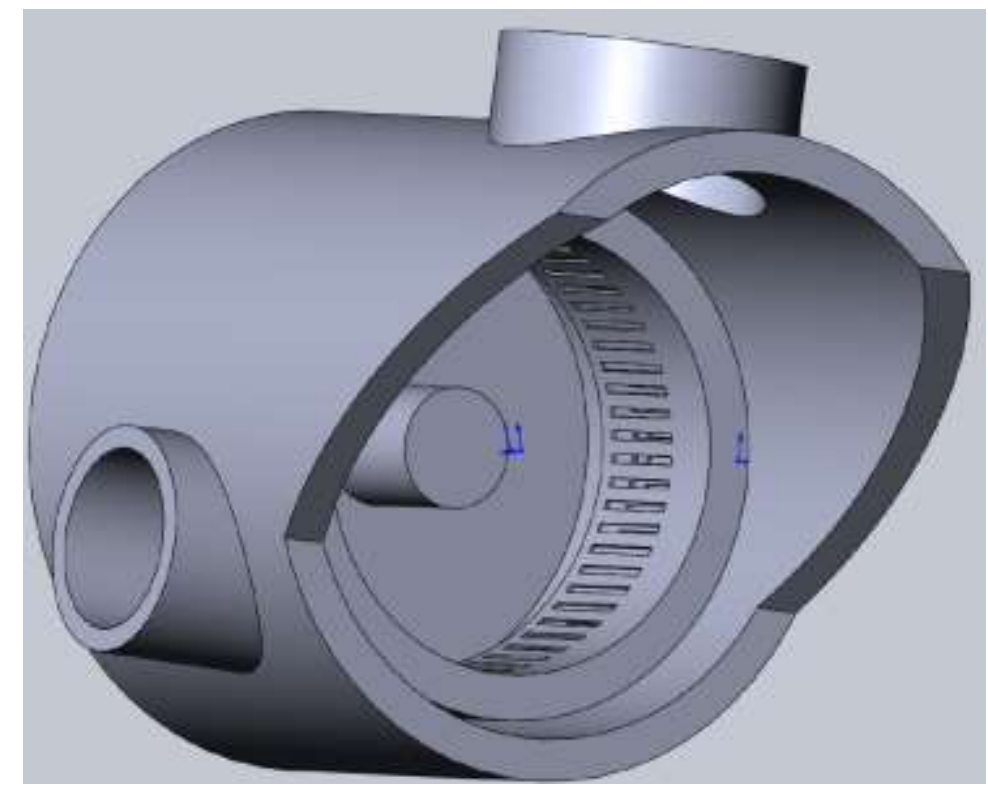

Figure 1. General view of the rotor-stator system in RPA

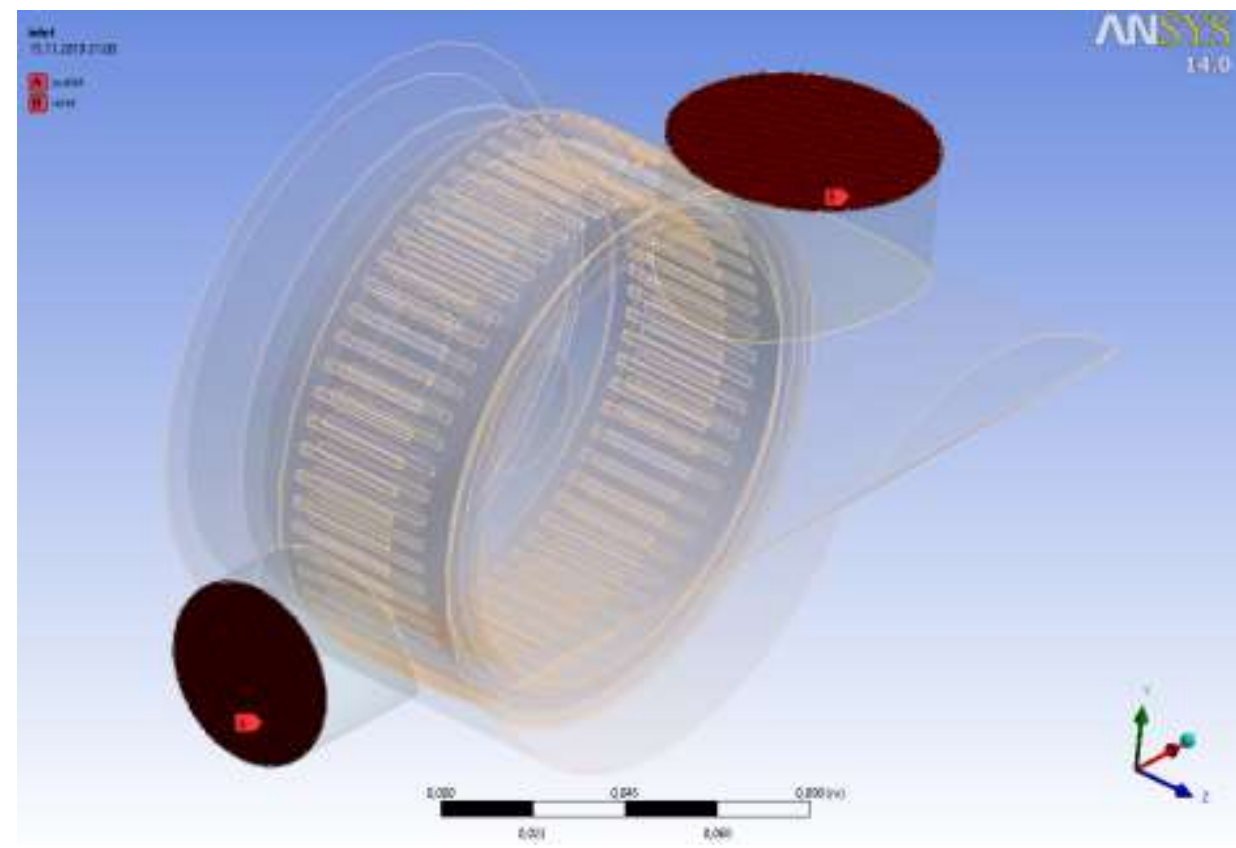

Figure 2. Boundary conditions in RPA channels

The volume of RPA rotor is $3,798 \cdot 10^{-4} \mathrm{~m}^{3}$. The rotor, on the $\mathrm{z}$ axis, rotates at a speed of $3000 \mathrm{rpm}$. Numerical simulations were performed three times with a constant dynamic viscosity $0.2 ; 1.1$ and $2.1 \mathrm{~Pa}$.

For numerical modeling, a mixture of water and grain in the ratio $10 \times 1$ was introduced. Thermophysical properties of water, which varies with temperature, are 
taken from the relevant tables. Thermophysical properties of grain are considered independent of temperature and equal to:

- Density $770 \mathrm{~kg} / \mathrm{m}^{3}$;

- The thermal conductivity coefficient $0.11 \mathrm{~W} /\left(\mathrm{m}^{0} \mathrm{C}\right)$;

- Heat capacity $1550 \mathrm{~kJ} /\left(\mathrm{kg}^{0} \mathrm{C}\right)$

The mixture of water/grain (Y), for all required thermophysical quantities, is calculated by the following formula:

$$
Y=\frac{10 \cdot x_{1}+1 \cdot x_{2}}{x_{1}+x_{2}}
$$

where $x$ is the thermophysical quantity, index 1 is the value of water, 2 is the value of grain. The thermophysical properties of the liquid grain mixture depend on the temperature.

Thermophysical properties of the feed mixture are presented in Table 1 .

Table 1

Thermophysical properties of the mixture

\begin{tabular}{|c|c|c|c|}
\hline $\begin{array}{c}\text { Temperature, } \\
{ }^{\circ} \mathrm{C}\end{array}$ & Density, $\mathrm{kg} / \mathrm{m}^{3}$ & $\begin{array}{c}\text { Heat capacity, } \\
\mathrm{kJ} /\left(\mathrm{kg} \cdot{ }^{\circ} \mathrm{C}\right)\end{array}$ & $\begin{array}{c}\text { Thermal conductivity } \\
\text { coefficient } \\
\mathrm{W} /\left(\mathrm{m} \cdot{ }^{\circ} \mathrm{C}\right)\end{array}$ \\
\hline 20 & 977.5364 & 3933.63636 & 0.55454545 \\
\hline 30 & 975.1909 & 3939.09091 & 0.57181818 \\
\hline 40 & 972.0455 & 3940.0 & 0.58727273 \\
\hline 50 & 968.2 & 3941.81818 & 0.59909091 \\
\hline 60 & 963.7545 & 3945,45455 & 0.60909091 \\
\hline 70 & 958.7545 & 3950.0 & 0.61727273 \\
\hline 80 & 953.2727 & 3955.45455 & 0.62272727 \\
\hline 90 & 947.3273 & 3963.63636 & 0.62636364 \\
\hline
\end{tabular}

\subsubsection{Using the finite element method to build a 3D mesh in ANSYS Meshing}

The finite element method (FEM) is used to numerically calculate the problems of hydrodynamics and heat transfer. In English literature it is called Finite Elements 
Method (FEM). The essence of the method is an approximate solution of the variational problem. To formulate this problem, we use the concept of functionality. The operator I $[f(x)]$ is called a functional that is given on some set of functions, if for each function $f(x)$ a certain numerical value of I [ $f(x)$ ] is matched [248]. In other words, a functional that is a "function from a function." Often functionals have the form of integrals. The variational problem is to find a function $\mathrm{f}(\mathrm{x})$ that corresponds to the minimum value of the functional I $[\mathrm{f}(\mathrm{x})]$. The appearance of this functionality is different for different tasks and is selected by special choice.

Currently, FEM has found wide application in solving problems of thermal conductivity in solids and in the calculation of materials for strength. In addition, this method is used to calculate the flow of liquids and gases [247]. There are also methods that combine elements of the finite volume method and the finite element method $[249,250]$. The combination of these methods allows the use of a wider range of computational grids (tetragonal grids, pyramidal, prismatic, polyhedral), which is necessary when solving problems with complex geometry. This approach is used by CFD packages Ansys CFX, Ansys Fluent, Star-CD, Star-CCM +, Comsol and others.

The ANSYS package has many applications for almost all fields of modern science and technology, including agriculture. It should be borne in mind that different physical tasks require different approaches to modeling and creating a calculation grid. Consider some features of the construction of the grid for problems of hydrodynamics, heat and mass transfer - its creation in ANSYS Meshing, in particular 2D. ANSYS Meshing is well adapted to create a grid for CFD applications ANSYS - CFX or FLUENT, but keep in mind that the general logic of building a grid can be transferred to other programs, including programs that are not used in ANSYS.

The grid was built in the ANSYS Meshing grid generator on the Workbench platform. In ANSYS Meshing Application there are the following breakdown methods for 3D geometry:

- Automatic;

- Methods of constructing a tetrahedron grid (Tetrahedrons):

- on the basis of a surface grid (Patch Conforming); 
- independent of surfaces (Patch Independent);

- CFX-Mesh;

- Methods of constructing a hexahedral grid:

- broach (Sweep);

- broach for shell bodies (Thin sweep);

- Multi Zone;

- multi-zone (based on ICEM CFD block grid);

- with a predominance of hexahedrons (Hex Dominant).

Software packages use several basic types of grid for 3D: tetrahedral, Cartesian and hexahedral. The tetrahedral grid allows to create the cells close in the form to borders of settlement area and to zones with big gradients of speeds and temperatures that allows to model boundary layers. At the same time, creating a tetrahedral grid is a very time consuming process. Cartesian grid allows only rectangular cells to be created, which can worsen the solutions of the boundary layer equations, but Cartesian grid is easier to create. There are a number of ways to solve the transfer equation in the region of boundary layers in the presence of high gradients of flow parameters.

The hexahedral grid, to solve the problem with the same accuracy, will contain more than 2 times fewer nodes, compared to the tetrahedral grid. Therefore, its application requires fewer elements to solve the problem of CFD.

Anisotropic elements can be combined with anisotropic geometry (boundary layers, areas with large curvature and long finite elements). For arbitrary configurations, the hexahedral grid requires a lot of preparatory operations, but this leads to a better result. For many simpler broach configurations, grids can be created more easily and quickly using sweep and multi-zone construction.

In the table 3.4 shows the settings and mesh results in ANSYS Meshing for further transfer of the calculation of heat and mass transfer and hydrodynamics in ANSYS Fluent. Geometry is built in real size. The number of elements and faces is quite large (Table 2). Due to the large size of the RPA, the size of the element and the face is not increased much due to limited production and design power of the computer. 
In Fig. 3-5 shows the constructed RPA mesh. The maximum mesh size for the entire volume does not exceed $1 \cdot 10^{-3} \mathrm{~m}$. These measures are used to improve the calculation of hydrodynamics. In the section YZ and YX (Fig. 4) the thickening of the mesh in the grooves of the rotor and stator is clearly visible.

Table 2

Mesh construction parameters for RPA

\begin{tabular}{|c|c|}
\hline Parameter & Value \\
\hline Mesh quality indicator (orthogonal quality) & 0.161 \\
\hline Number of elements, pcs & 4485359 \\
\hline Number of nodes, pcs & 4791394 \\
\hline Curvature angle, city & 45 \\
\hline Method & Cut-Cell \\
\hline Maximum size, $\mathrm{m}$ & $1 \cdot 10^{-3}$ \\
\hline Minimum size, $\mathrm{m}$ & $5 \cdot 10^{-4}$ \\
\hline
\end{tabular}

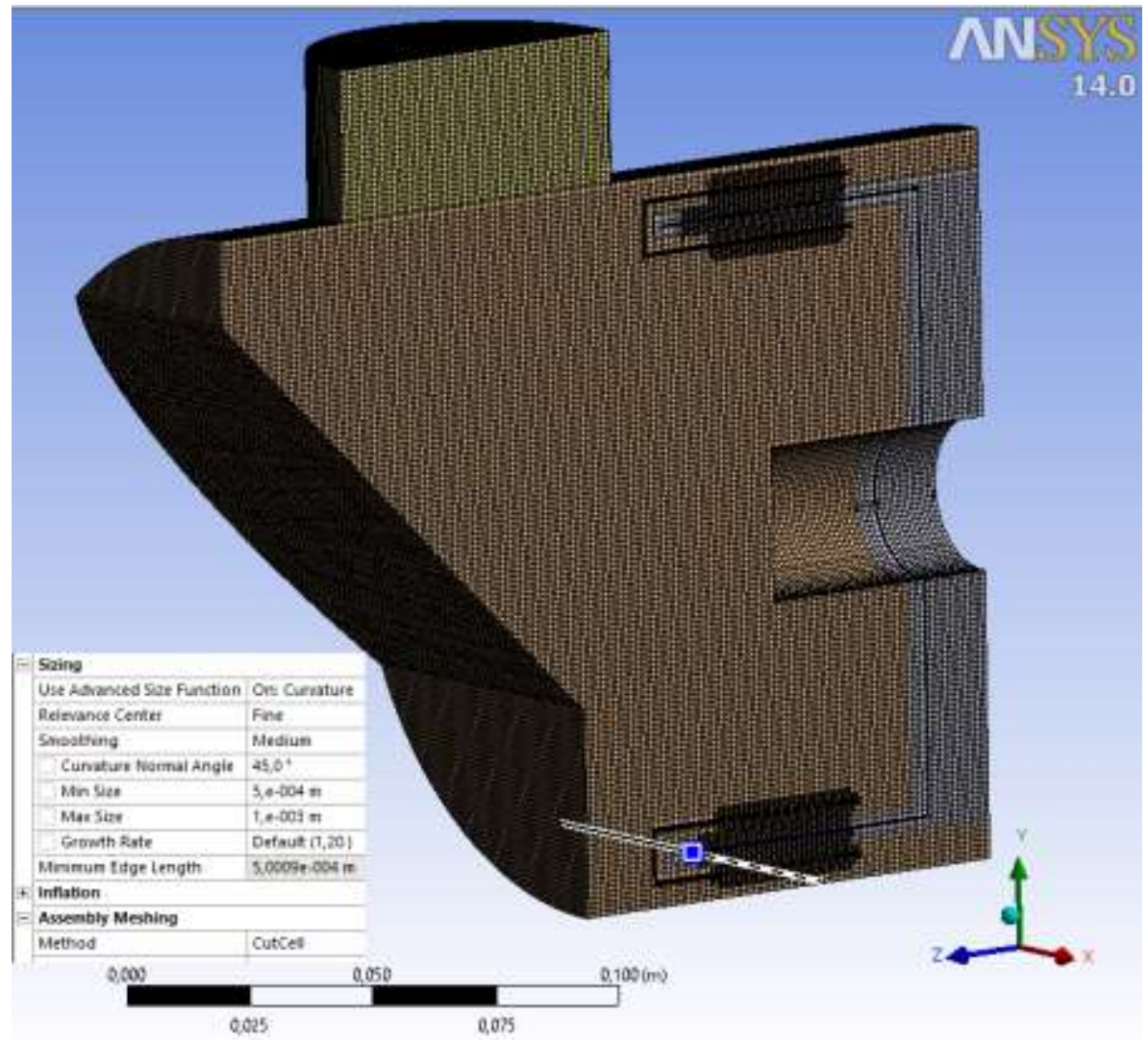

Figure 3. The result of the constructed mesh in the longitudinal section YZ 


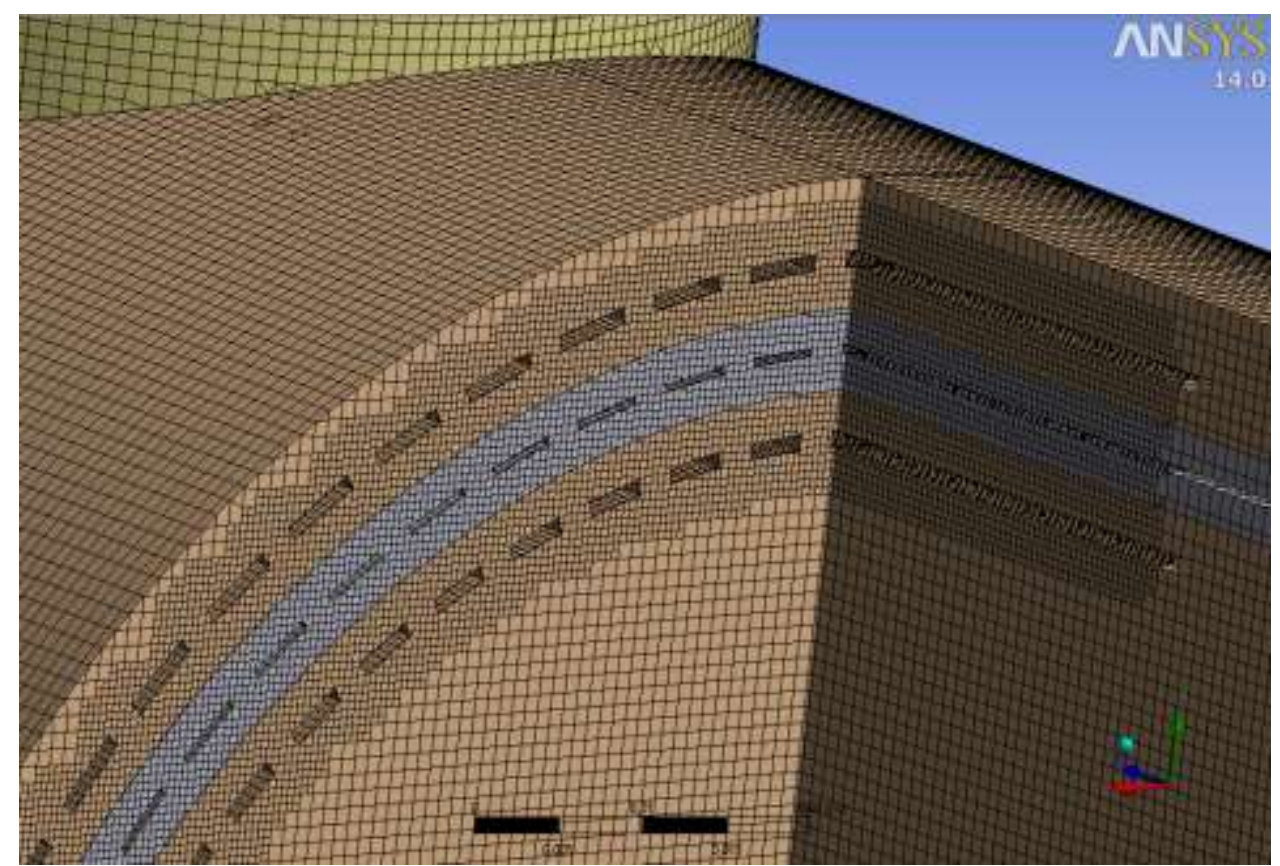

Figure 4. The result of the constructed mesh in the section of $\mathrm{YZ}$ and $\mathrm{YX}$

The size of the thickening of the mesh near the grooves is $5 \cdot 10^{-4} \mathrm{~m}$ (Fig. 5). Thanks to which you can better assess the quality and shortcomings of the grid itself. The number of mesh elements is about 4.5 million. The quality of the mesh in orthogonal quality is 0.161 . Detailed results are given in Table 2 .

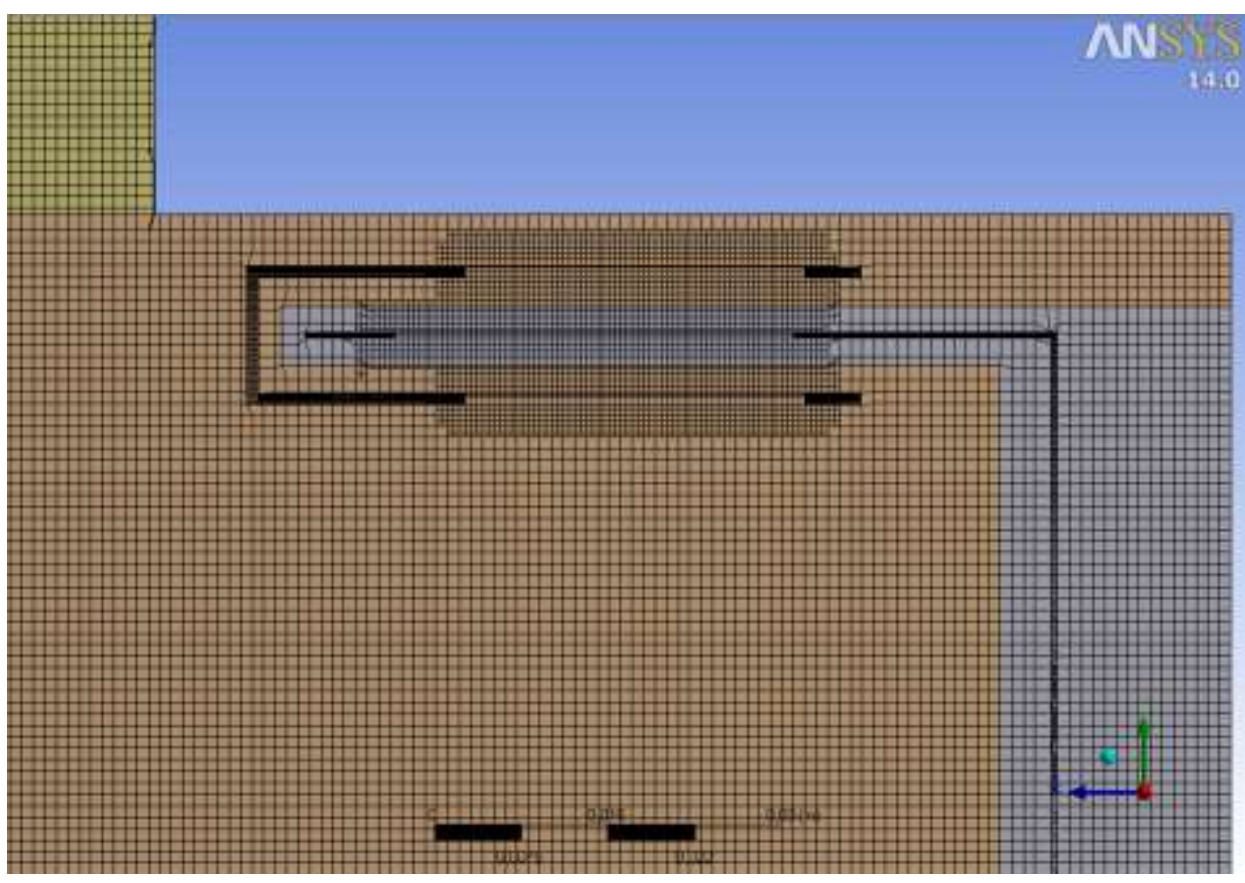

Figure 5. The result of the constructed mesh between the grooves in the longitudinal section YZ 
Using the finite element method, a 3D calculation grid in ANSYS Meshing software package for solving hydrodynamics and heat transfer problems in RPA was constructed. As a result of building various grids for CFD models, the most optimal and high-quality one was chosen, which allows to obtain reliable and most accurate results of heat and mass transfer processes in RPA.

\subsubsection{Mathematical modeling of heat and mass transfer processes in RPA}

The motion of a viscous liquid or gas is described by a system of equations, which includes the equation of continuity and the equation of conservation of momentum in projections on the coordinate axis. If the motion of the medium is accompanied by heat transfer, the equation of energy conservation is added to the system of these equations.

The mathematical model is based on the Navier-Stokes equation [251] and the energy transfer equation for convective flows. The standard $k-\varepsilon$ turbulence model was used in the calculations [252]. The initial equations are given below.

Navier-Stokes equations:

$$
\left.\begin{array}{l}
\rho\left(\frac{\partial u}{\partial t}+u \frac{\partial u}{\partial x}+v \frac{\partial u}{\partial y}+w \frac{\partial u}{\partial z}\right)=\rho g_{x}-\frac{\partial p}{\partial x}+\mu\left(\frac{\partial^{2} u}{\partial x^{2}}+\frac{\partial^{2} u}{\partial y^{2}}+\frac{\partial^{2} u}{\partial z^{2}}\right), \\
\rho\left(\frac{\partial v}{\partial t}+u \frac{\partial v}{\partial x}+v \frac{\partial v}{\partial y}+w \frac{\partial v}{\partial z}\right)=\rho g_{y}-\frac{\partial p}{\partial y}+\mu\left(\frac{\partial^{2} v}{\partial x^{2}}+\frac{\partial^{2} v}{\partial y^{2}}+\frac{\partial^{2} v}{\partial z^{2}}\right), \\
\rho\left(\frac{\partial w}{\partial t}+u \frac{\partial w}{\partial x}+v \frac{\partial w}{\partial y}+w \frac{\partial w}{\partial z}\right)=\rho g_{z}-\frac{\partial p}{\partial z}+\mu\left(\frac{\partial^{2} w}{\partial x^{2}}+\frac{\partial^{2} w}{\partial y^{2}}+\frac{\partial^{2} w}{\partial z^{2}}\right),
\end{array}\right\}
$$

where $\rho$ is the density of the medium, $\mathrm{kg} / \mathrm{m}^{3} ; \mu$ - dynamic viscosity of the medium, $\mathrm{Pa}$ - $\mathrm{s} ; \mathrm{p}$ - pressure, Pa; $\mathrm{u}, \mathrm{v}, \mathrm{w}$, - vector field of velocities, $\mathrm{m} / \mathrm{s} ; \mathrm{t}$ - time, $\mathrm{s}$.

Continuity equation:

$$
\frac{\partial \rho}{\partial \tau}+\frac{\partial(u \rho)}{\partial x}+\frac{\partial(v \rho)}{\partial y}+\frac{\partial(w \rho)}{\partial z}=0,
$$

Energy conservation equation:

$$
\rho C_{p}\left(\frac{\partial T}{\partial \tau}+u \frac{\partial T}{\partial x}+v \frac{\partial T}{\partial y}+w \frac{\partial T}{\partial z}\right)=\frac{\partial}{\partial x}\left(\lambda \frac{\partial T}{\partial x}\right)+\frac{\partial}{\partial y}\left(\lambda \frac{\partial T}{\partial y}\right)+\frac{\partial}{\partial z}\left(\lambda \frac{\partial T}{\partial z}\right)+Q(\tau, x, y, z) .
$$


where $\mathrm{T}$ is the temperature at some point, $\mathrm{K} ; \lambda$ is the thermal conductivity of the medium, $\mathrm{Wt} / \mathrm{m} \bullet \mathrm{K} ; \mathrm{C}_{\mathrm{p}}$ - specific heat of the medium, $\mathrm{J} / \mathrm{kg} \bullet \mathrm{K}$.

\section{Turbulence model}

For the flow of coolants in the channels it is necessary to choose a model of turbulence that corresponds to the conditions of hydrodynamic flow in these channels. One of the most common is the standard k- $\varepsilon$ model used in ANSYS FLUENT software package, used in practical engineering calculations of flows in channels and was proposed by Launder and Spalding [252]. The reliability, economy and accuracy of this model for a wide range of turbulent flows explains its wide application for modeling heat transfer in channels of different configurations.

The standard k- $\varepsilon$ [252] model is based on the model of the turbulence transfer equation of kinetic energy $(\mathrm{k})$ and its dissipation rate $(\varepsilon)$. The equation of the transport model for $\mathrm{k}$ is from the exact equation, and the equation of the transport model for $\varepsilon$ is obtained for physical reasons.

In deriving the $\mathrm{k}-\varepsilon$ model, the flow is assumed to be completely turbulent and the effects of molecular viscosity are negligible, ie the standard k- $\varepsilon$ model is used for fully turbulent flows.

Subsequently, the standard k- $\varepsilon$ model was improved, which increased its efficiency. There are two improved versions of this model, which are available in the software package ANSYS FLUENT, namely, RNG k- $\varepsilon$ model [254] and implemented (realizable) k- $\varepsilon$ model [253].

The transfer equation of the standard $k-\varepsilon$ model.

The kinetic energy of turbulence $\mathrm{k}$ and its dissipation rate $\varepsilon$ is found from the solution of the following transport equations [255-257]:

$$
\begin{gathered}
\frac{\partial}{\partial t}(\rho k)+\frac{\partial}{\partial x_{i}}\left(\rho k u_{i}\right)=\frac{\partial}{\partial x_{j}}\left[\left(\mu+\frac{\mu_{t}}{\sigma_{k}}\right) \frac{\partial k}{\partial x_{j}}\right]+G_{k}+G_{b}-\rho \varepsilon-Y_{M}+S_{k} \\
\frac{\partial}{\partial t}(\rho \varepsilon)+\frac{\partial}{\partial x_{i}}\left(\rho \varepsilon u_{i}\right)=\frac{\partial}{\partial x_{j}}\left[\left(\mu+\frac{\mu_{t}}{\sigma_{\varepsilon}}\right) \frac{\partial \varepsilon}{\partial x_{j}}\right]+G_{1 \varepsilon} \frac{\varepsilon}{k}\left(G_{k}+G_{3 \varepsilon} G_{b}\right)-C_{2 \varepsilon} \rho \frac{\varepsilon^{2}}{k}+S_{\varepsilon}
\end{gathered}
$$


where, $G_{k}$ is the generation of kinetic energy turbulence due to velocity gradients (see equation (12)); $\mathrm{G}_{\mathrm{b}}$ is the generation of kinetic energy turbulence (see equation (15)); $\mathrm{Y}_{\mathrm{M}}$ is the contribution to the expansion of the compressibility fluctuations of turbulent flows in the total dissipation rate (see equation (18)); $\mathrm{C}_{1 \mathrm{e}}, \mathrm{C}_{2 \mathrm{e}}$ and $\mathrm{C}_{3 \mathrm{e}}$ - are constants; $\sigma \mathrm{k}$ and $\sigma \mathrm{e}$ are Prandtl turbulent numbers for $\mathrm{k}$ and $\varepsilon$, respectively. The values of $S_{\mathrm{k}}, S_{\mathrm{e}}$ - are defined as the characteristics of the kinetics of kinetic energy and the dissipation of this energy:

$$
\begin{gathered}
S_{k}=\tau_{i j} \frac{\partial u_{i}}{\partial x_{j}}-\rho \varepsilon-\mu_{t}\left(\frac{g_{i} \partial \rho}{\sigma_{B} \rho \partial x_{i}}\right) \\
S_{\varepsilon}=C_{\varepsilon 1} \frac{\varepsilon}{k}\left(f_{1} \tau_{i j} \frac{\partial u_{i}}{\partial x_{j}}+\mu_{t} C_{B}\left(\frac{g_{i} \partial \rho}{\sigma_{B} \rho \partial x_{i}}\right)\right)-C_{\varepsilon 2} f_{2} \frac{\rho \varepsilon^{2}}{k}
\end{gathered}
$$

where $\mathrm{C}_{\varepsilon 1}=1.44, \mathrm{C}_{\varepsilon 2}=1.92, \sigma_{\varepsilon}=1.3, \sigma_{\mathrm{k}}=1$ - empirical coefficients [257]; $\mathrm{f}_{1}, \mathrm{f}_{2}$ variables that depend on the coefficients of dynamic and turbulent viscosity and are determined by the following formulas:

$$
f_{1}=1+\left(\frac{0,05}{f_{\mu}}\right)^{3}, \quad f_{2}=1-e^{-R_{T}^{2}} .
$$

The values of constants and parameters in this model were determined from experiments for fundamental turbulent flows, including shear flows, boundary layers, mixed layers, as well as for the decomposition of an isotropic turbulence grid. They have proven to be quite effective for a wide range of wall-bound landslides.

Modeling of turbulent viscosity.

Turbulent (or vortex) viscosity $\mu \mathrm{t}$ 'is calculated through $\mathrm{k}$ and $\varepsilon$ as follows:

$$
\mu_{t}=\rho C_{\mu} \frac{k^{2}}{\varepsilon}
$$

where, $\mathrm{C}_{\mu}$ is a constant.

The degree to which $\varepsilon$ depends on buoyancy is determined by the constant $\mathrm{C}_{3 \mathrm{e}}$. In ANSYS FLUENT, the value of $\mathrm{C}_{3 \mathrm{e}}$ is calculated by the following ratio [258]:

$$
C_{3 e}=\tanh \left|\frac{v}{u}\right|
$$


where $v$ is the component of the flow velocity that is parallel to the gravitational vector, $u$ is the component of the flow velocity perpendicular to the gravitational vector. Thus, $\mathrm{C}_{3 \mathrm{e}}$ will be equal to 1.0 for floating shear layers, for which the main flow direction is combined with the direction of gravity. For floating shear layers that are perpendicular to the gravitational vector, $\mathrm{C}_{3 \mathrm{e}}$ becomes zero.

Modeling of turbulent processes in the $k-\varepsilon$ model.

The parameter $\mathrm{G}_{\mathrm{k}}$, is a process of kinetic energy of turbulence, which is modeled equally for standard, RNG and realizable k- $\varepsilon$ models and is defined as:

$$
G_{k}=-\rho \overline{u_{i}^{\prime} u_{j}^{\prime}} \frac{\partial u_{j}}{\partial x_{i}}
$$

To estimate $\mathrm{G}_{\mathrm{k}}$ according to Bussinesc's hypothesis:

$$
G_{k}=\mu_{t} S^{2}
$$

where $S$ is the modulus of the average rate of change of the strain tensor, defined as:

$$
S \equiv \sqrt{2 S_{j} S_{/ j}}
$$

Effects of the effect of buoyancy on turbulence in the $k-\varepsilon$ model

Generation of turbulence due to buoyancy is determined by:

$$
G_{b}=\beta g_{i} \frac{\mu_{t}}{\operatorname{Pr}_{t}} \frac{\partial T}{\partial x_{i}}
$$

where Pr is the turbulent Prandtl number for the energy and $g_{i}$ and the component of the gravitational vector in the $i$-th direction. For standard $\mathrm{k}-\varepsilon$ models, its value is $\operatorname{Pr}=$ 0.85 .

The coefficient of thermal expansion $\beta$ is defined as:

$$
\beta=-\frac{1}{\rho}\left(\frac{\partial \rho}{\partial T}\right)_{p}
$$

For ideal gases, the ratio (15) is as follows:

$$
G_{b}=-g_{i} \frac{\mu_{t}}{\rho \operatorname{Pr}_{t}} \frac{\partial \rho}{\partial x_{i}}
$$

As can be seen from the transfer equations for the parameter $\mathrm{k}$ (see equation (5)), the turbulence of the kinetic energy is usually increased $\left(\mathrm{G}_{\mathrm{b}}>0\right)$ under conditions of 
unstable flow stratification. For stable stratification we have a reduction of this turbulence $\left(\mathrm{G}_{\mathrm{b}}<0\right)$. In ANSYS FLUENT package, the effects of buoyancy on the generation $\mathrm{k}$ are always taken into account if there is a non-zero gravitational field and a non-zero temperature gradient.

To account for these effects in the k- $\varepsilon$ model in ANSYS FLUENT, we use the scattering parameter of the extension $\mathrm{YM}$, which is included in k equation (6). This parameter is modeled according to Sarkar's proposal [259]:

$$
Y_{M}=2 \rho \varepsilon M_{t}^{2}
$$

where $\mathrm{M}_{\mathrm{t}}$ - is a turbulent Mach number, defined as:

$$
M_{t}=\sqrt{\frac{k}{a^{2}}}
$$

where $(a \equiv \sqrt{\gamma R T})$ is the speed of sound.

\subsubsection{Results of numerical modeling of transfer processes in RPA}

As a result of numerical calculations in ANSYS FLUENT application package, all dynamic and thermal characteristics of the liquid feed mixture during its passage through the rotor-stator system were obtained [260-265]. The results of the calculations are presented in Fig. 6-8.

In Fig. 6 shows the pressure distribution in the channels of the rotary-pulsation apparatus during the flow of the feed mixture. As shown by the analysis of the pressure distribution, the maximum pressure increase in the flow of the feed mixture is observed in the areas between the rotor and the stator. In these areas, the pressure increase may be $55 \mathrm{kPa}$ compared to the pressure at the inlet to the channel. This indicates a significant effect of the viscosity of the mixture on the change in pressure in the rotorstator channels, which is accompanied by the conversion of the kinetic energy of rotation of the rotor into the thermal energy of the feed mixture.

In Fig. 7 shows the speed distributions in the channels of the working area in rotor-stator system. The maximum values of flow rate of the feed mixture are observed in the channels between the rotor and stator, where these values can exceed $7 \mathrm{~m} / \mathrm{s}$. 
When the liquid leaves the rotor-stator channel the flow vorticity areas are formed, which turbulizes the flow in the channels and leads to intensive mixing of the grain mixture. The result is a homogeneous feed grain mixture.

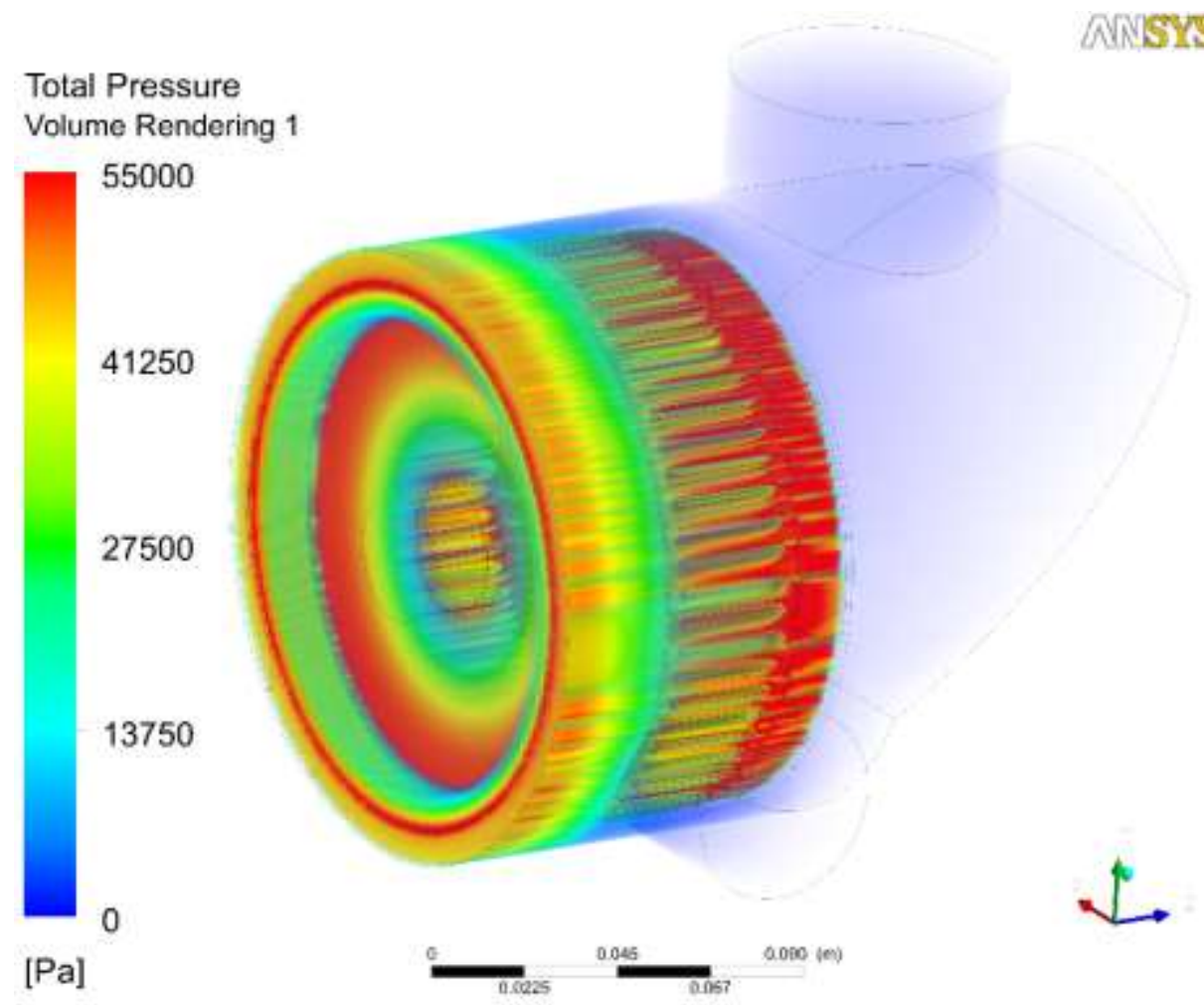

a)

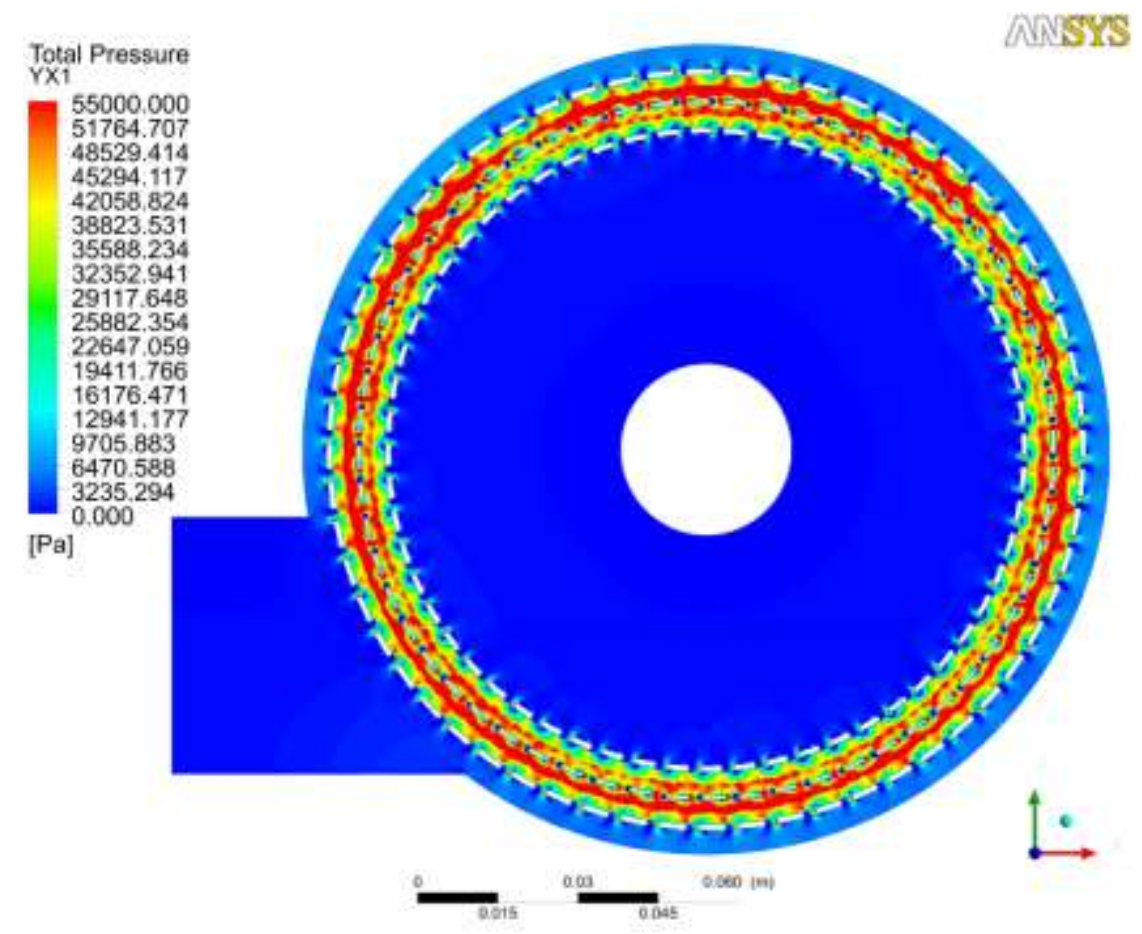

b) 


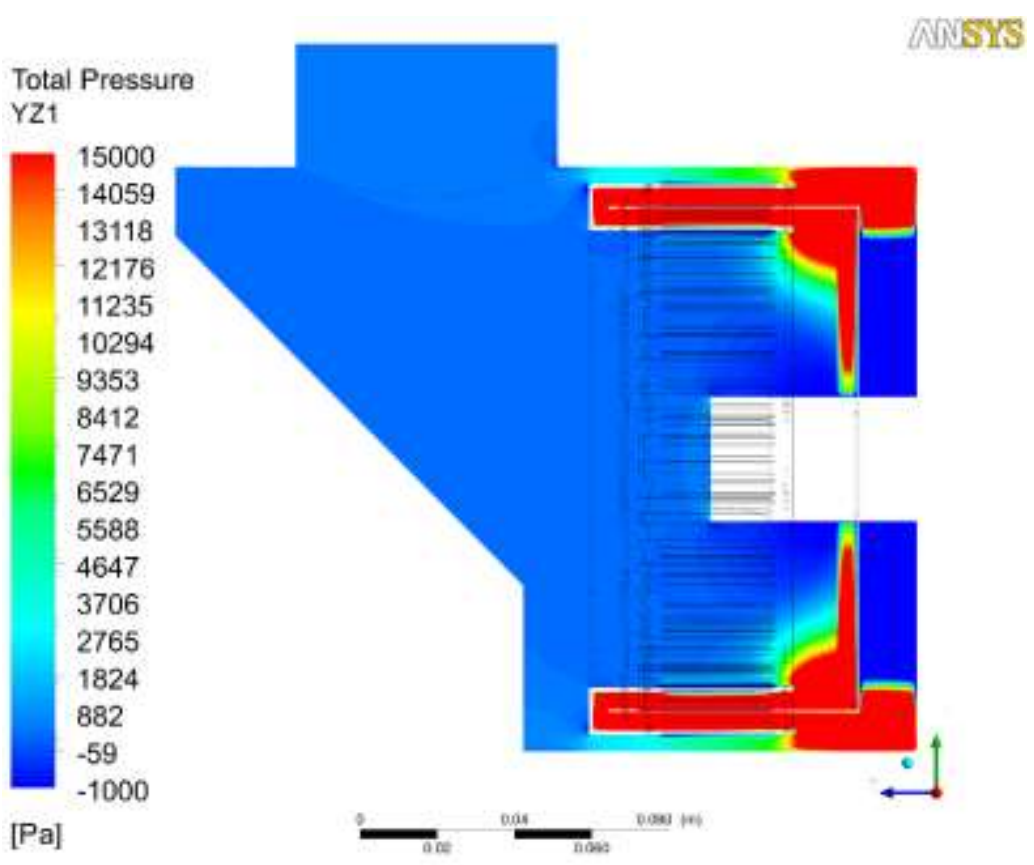

c)

Figure 6. Pressure distribution in the rotor-stator system: $a$ - 3D format; $b$ - cross section; $c$ - longitudinal section

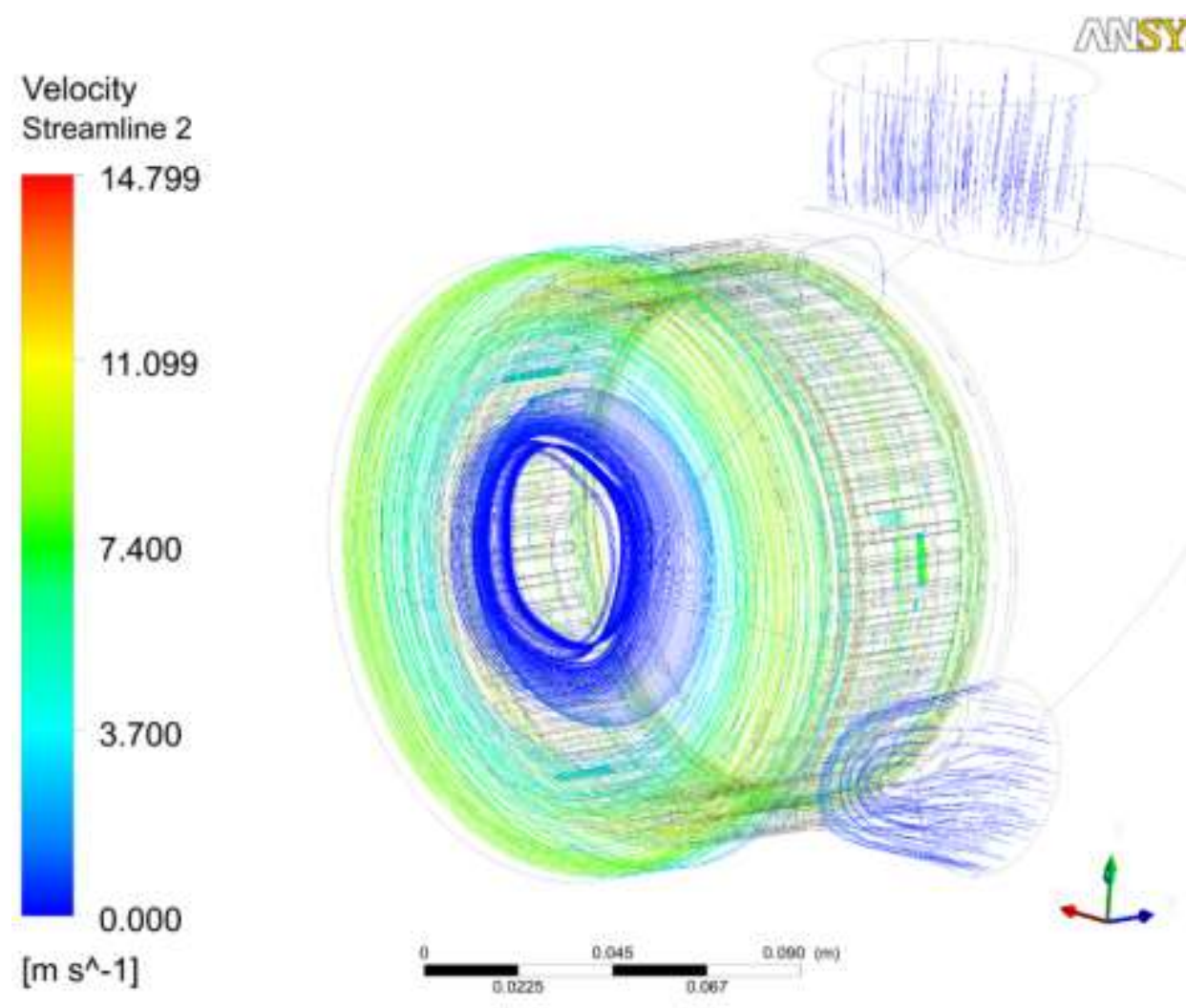

a) 


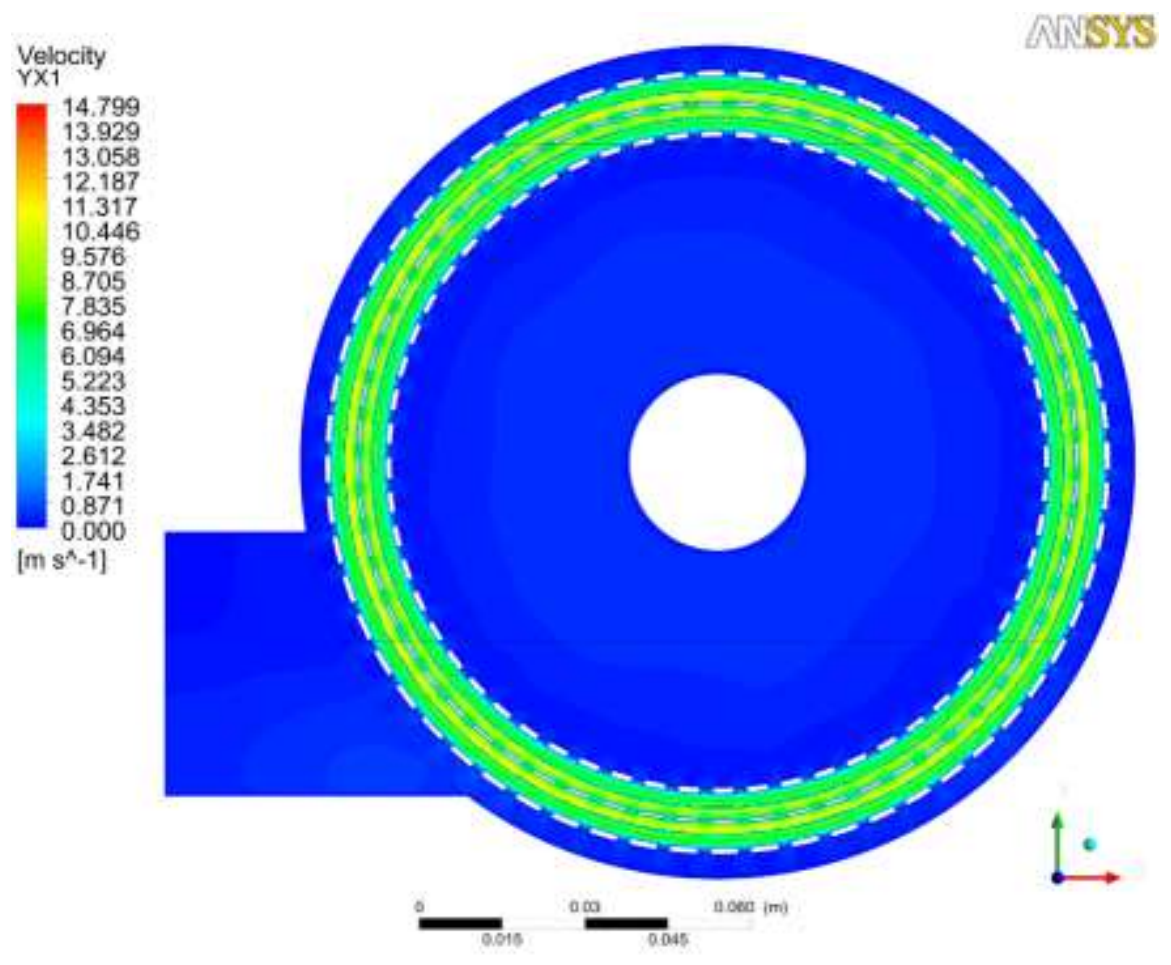

b)

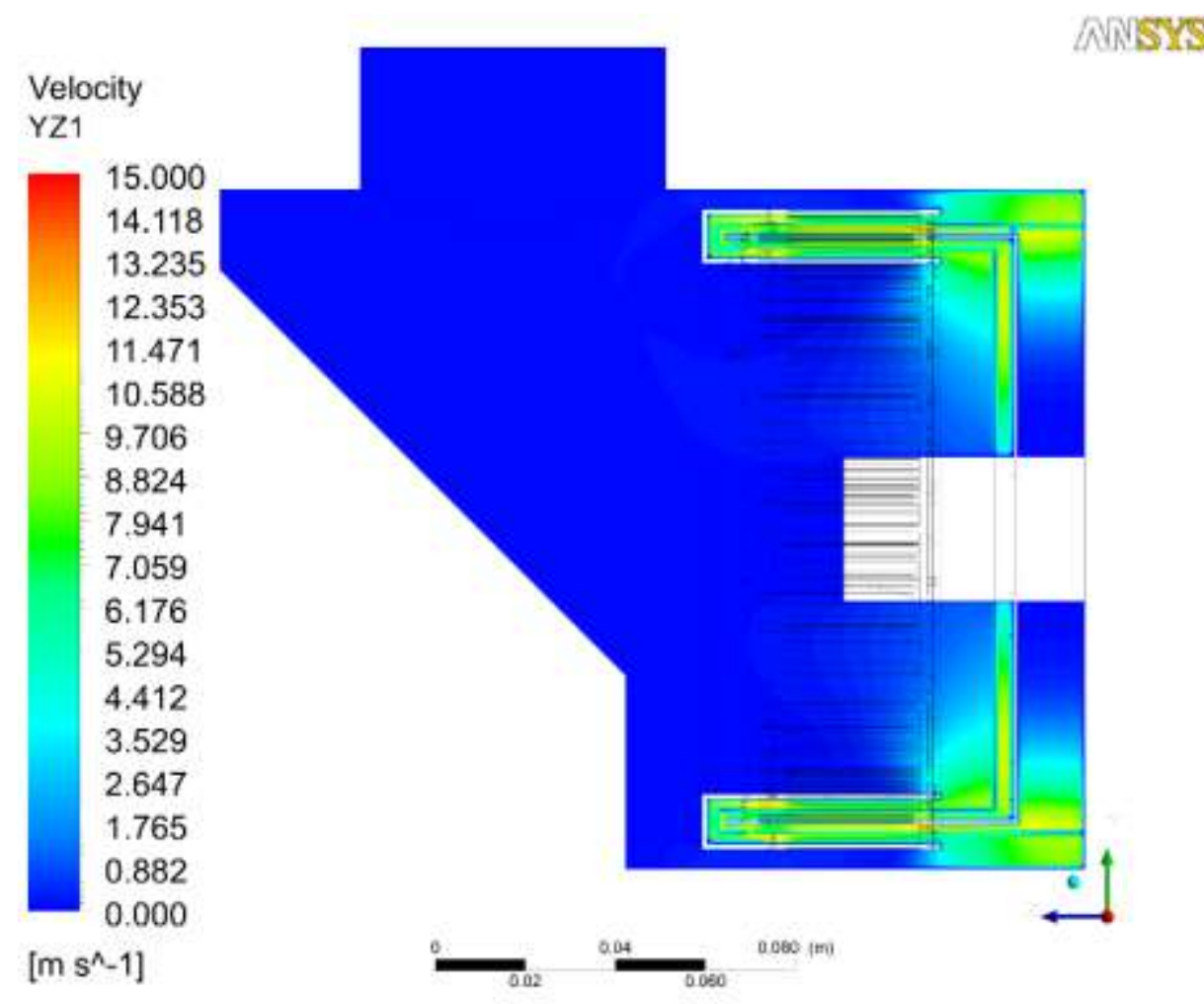

c)

Figure 7. Speed distribution in the rotor-stator system: $a$ - 3D format; $b$ - cross section; $c$ - longitudinal section 
Temperature distributions in the liquid mixture in the cross section of the channel are shown in Fig. 8. The highest values of temperatures in the liquid feed mixture take place in the areas adjacent to the surface of the end plane of the rotor and can exceed $21{ }^{0} \mathrm{C}$.

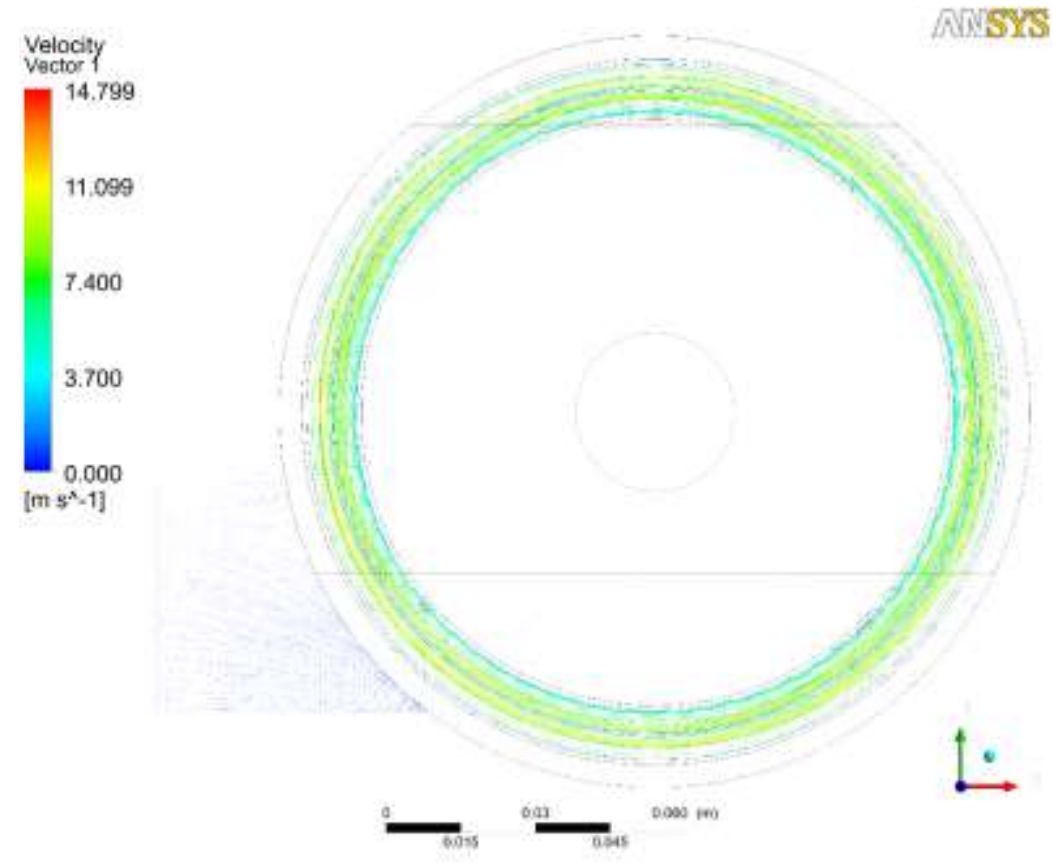

a)

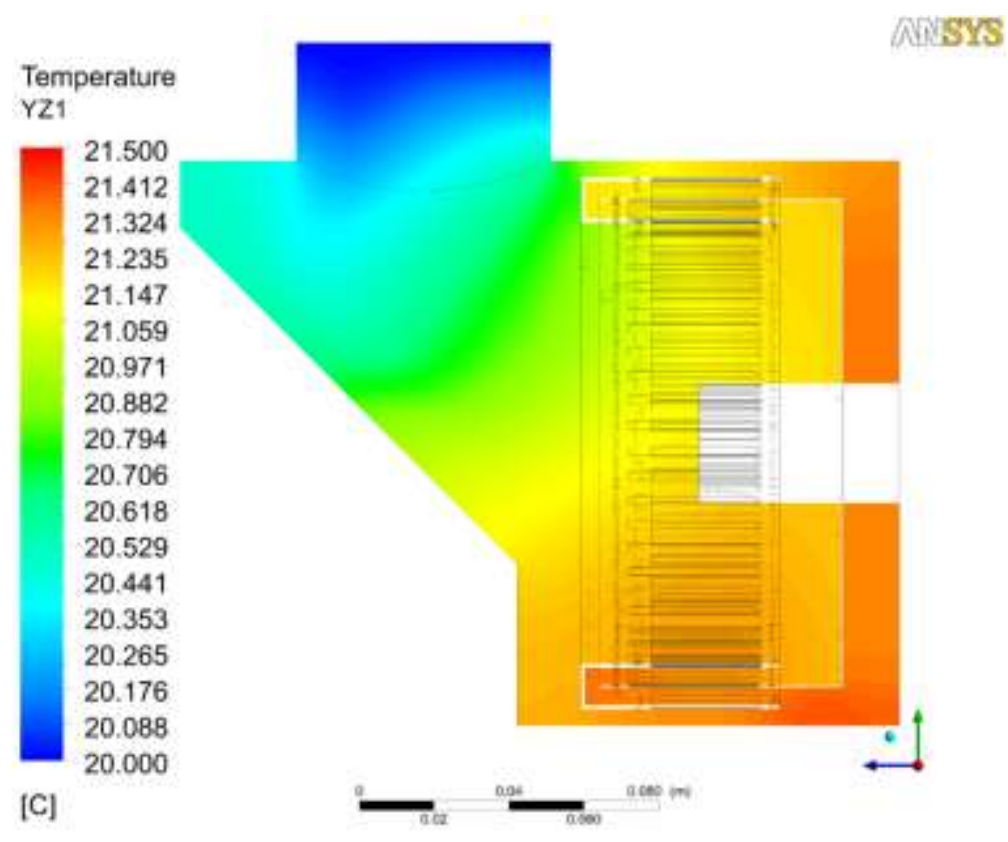

b)

Figure 8. Temperature distribution in the feed mixture for longitudinal (a) and cross

(b) cross section in the rotor-stator system 
This indicates a dissipation of the kinetic energy of rotation of the rotor and an increase in the temperature of the feed mixture during its processing. As a result, the feed mixture is heated, which is especially important in the winter and allows you not to heat the feed mixture during this period.

As a result of numerical simulation, the fields of pressures, velocities and temperatures in the liquid feed mixture were obtained, which made it possible to choose the design of the rotor-pulsation apparatus, which will be used to develop an experimental sample. such a device.

\subsubsection{Design and operation of experimental installation}

To study the process of processing starch-containing raw materials for the preparation of liquid feed, an experimental-industrial installation was developed and manufactured. This installation allows to study the nature of the heat and mass transfer processes in the processing of starch-containing medium. On the basis of the received experimental data it is possible to estimate reliability of theoretical researches, and also to work out technological process of preparation of liquid forages for farm animals.

Developed experimental installations provide: high efficiency of heat and mass transfer processes; dispersion, mixing, heating; ability to withstand operating stresses; tightness of detachable and non-detachable (welded) joints; safety and compliance with sanitary norms; convenience and reliability of service and repair. The experimental installation is presented in Fig. 9.

Experimental installation works on the following principle. According to the recipe of feed preparation, the grain mixture is fed into the receiver-dispenser, then the required amount of water is fed into the main hopper. After inclusion of the device in a stream of water flowing through the pipeline, grain moves and the mix is processed in RPA in the recirculation mode. The working chamber includes a rotor and a stator, which can have rectangular holes (Fig. 10). 


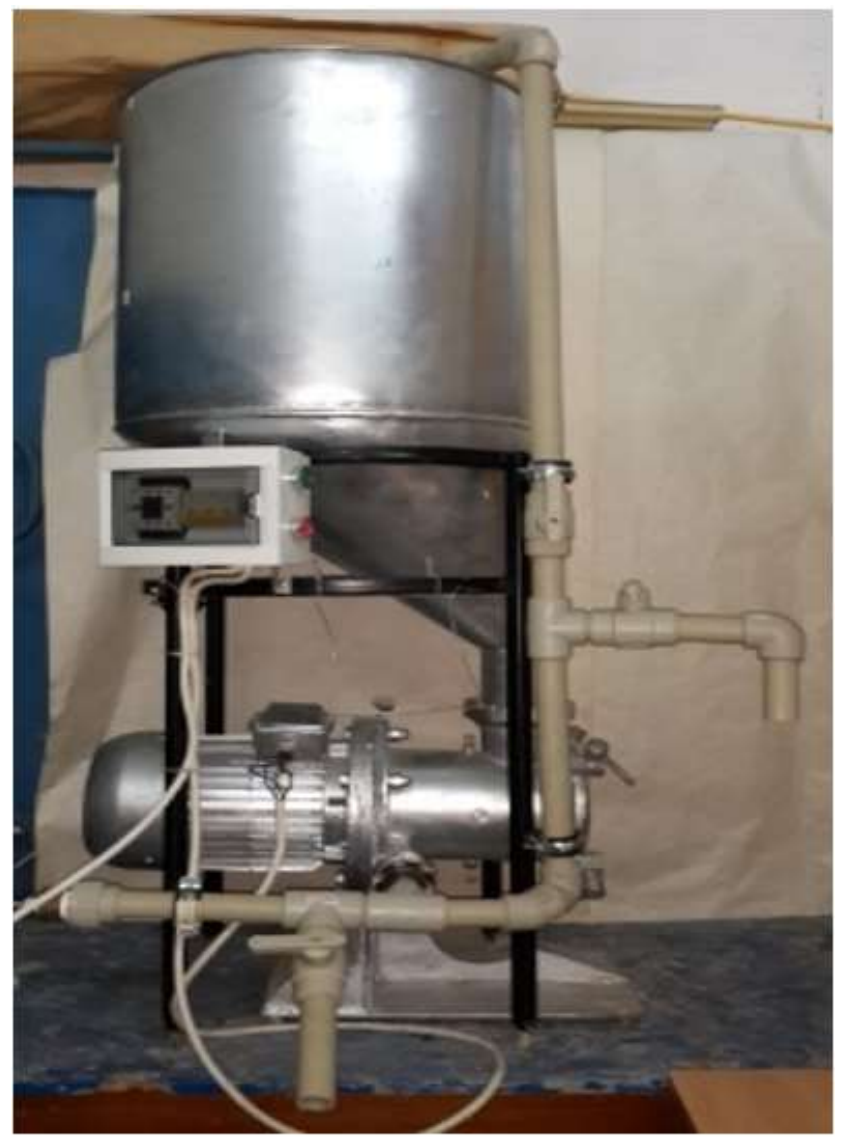

Figure 9. Rotary pulsation units for liquid feed preparation

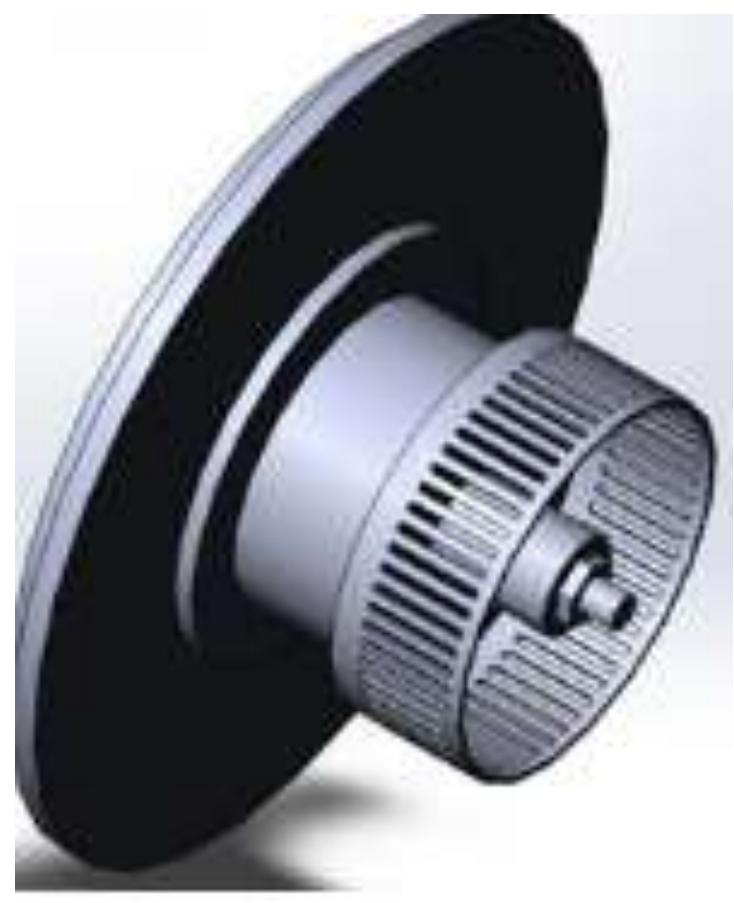

a)

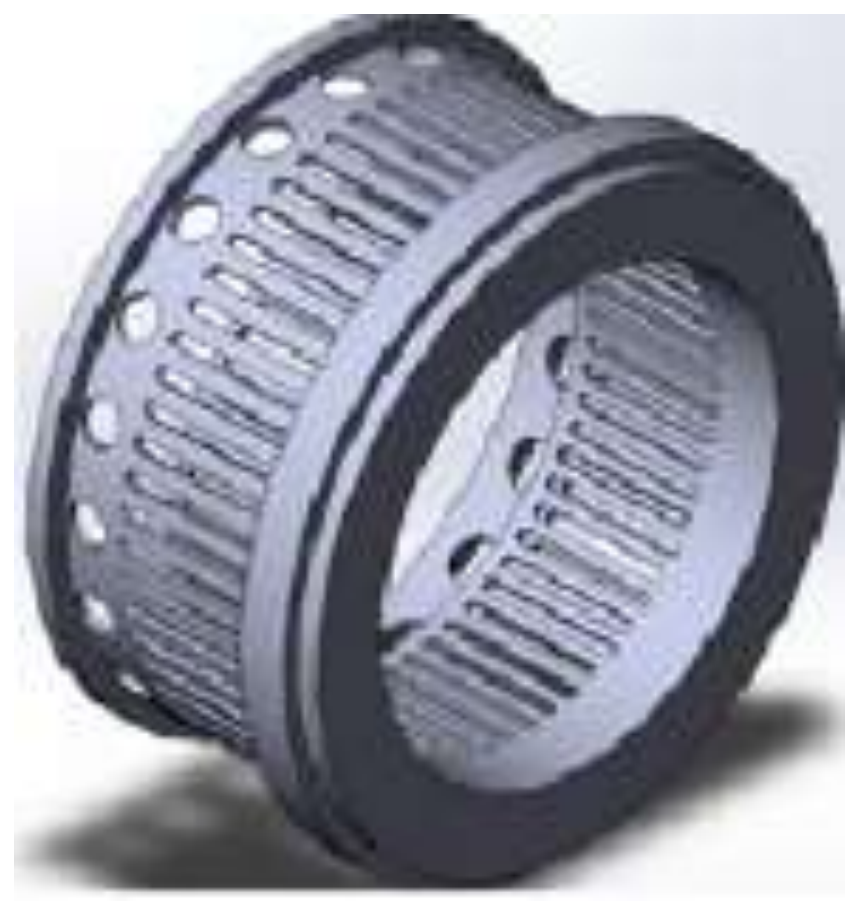

b)

Figure 10. General view of the rotor and stator in working chamber with rectangular holes: a) rotor; b) stator 
At such processing there is a grain crushing and its mixing with water. Since during the processing of the grain mixture it is heated due to energy dissipation, a bimetallic thermometer is provided on the hopper to monitor the temperature of the mixture. The scheme of the experimental setup is presented in Fig. 11.

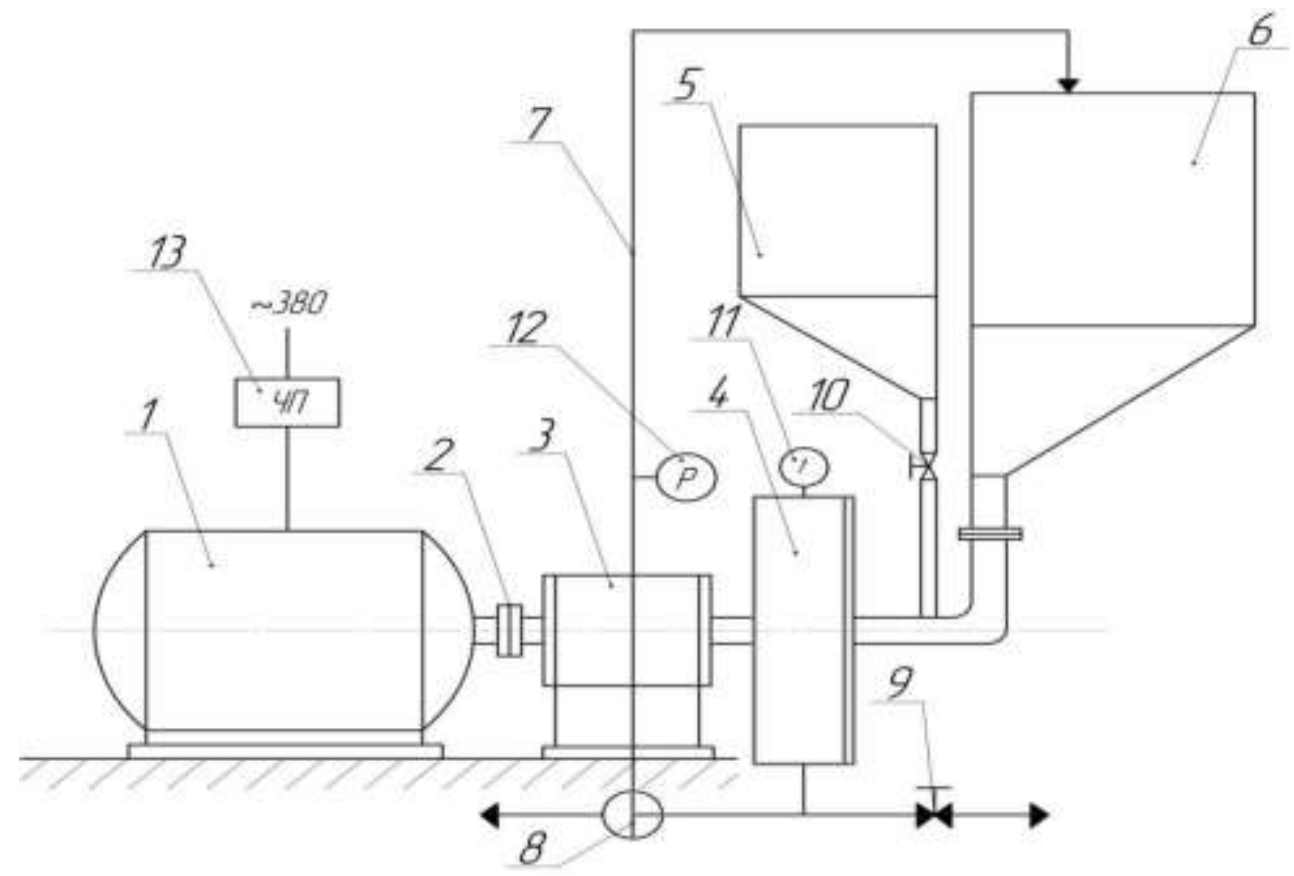

Figure 11. Schematic diagram of the experimental setup:

1 - electric motor; 2 - coupling; 3 - bearing assembly; 4 - RPA; 5 - receiving hopper for grain; 6 - bunker; 7 - recirculation pipeline; 8 - three-way crane; 9,10 - adjusting ball valves; 11 - bimetallic thermometer; 12 - manometer; 13 - frequency converter.

An experimental installation has been developed and manufactured which provides: high efficiency of heat and mass transfer processes; dispersion, mixing, heating; ability to withstand operating stresses; tightness of detachable and nondetachable (welded) joints; safety and compliance with sanitary norms; convenience and reliability of service and repair

Hydraulic characteristics: flow rate, pressure, as well as instrument readings were determined in a steady-state mode of operation of RPA at a given converter speed 
of the motor shaft. Temperature measurements were performed using measuring devices - bimetallic thermometer, manometer, vacuum gauge, wattmeter.

The electric motor 1 is designed to drive RPA rotor. In the control panel 13 the introductory automatic machine, the frequency converter, buttons of inclusion and switching off, signal armature are placed. Structurally RPA 4, mounted on a frame made of channel № 24. An electric motor is installed on the frame, connected to the bearing unit by means of a coupling. RPA is fixed on the flange of the bearing unit. The energy consumption of the electric motor was determined by a wattmeter.

\subsubsection{Experimental studies of feed preparation processes in RPA with rectangular holes.}

Experimental studies were performed for RPA with rectangular holes. In addition to studying the dynamics of changes in the average particle size of grain and the viscosity of the feed mixture at different times during its processing (see Section 4), parameters such as energy consumption and temperature of the mixture during its processing were determined. The last parameter is important, because the traditional methods of preparation of feed mixture based on dry feed requires additional energy costs for its heating, especially in winter.

\subsubsection{Determination of particle size distribution of liquid grain mixture}

The use of an electron microscope to determine the particle size distribution of the grain mixture during its processing makes it possible to observe how the average grain particle size changes depending on the processing time and the number of cycles of passage of the mixture through the working chamber. The main purpose of these studies is to determine the processing time and the number of cycles in which the composition of the mixture will be optimal in terms of maximum assimilation by domestic animals. At the same time, grain mixtures were studied using different types of grain - wheat, corn, barley. 
The results of research to determine the average particle size of grain as a function of time are shown in Fig. 12. Studies were performed for different grain types at different grain concentrations in the liquid grain mixture.

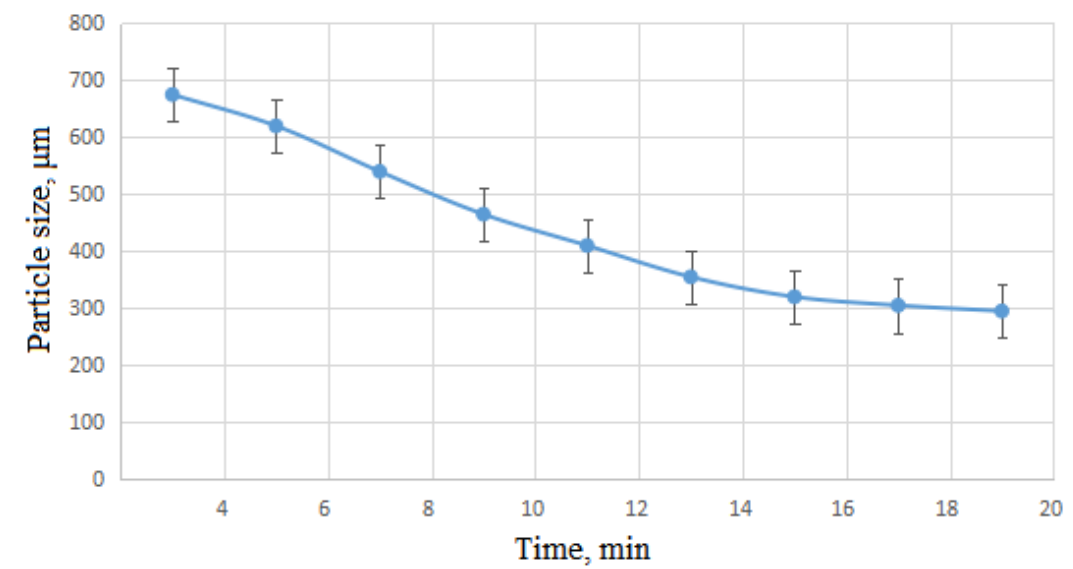

a)

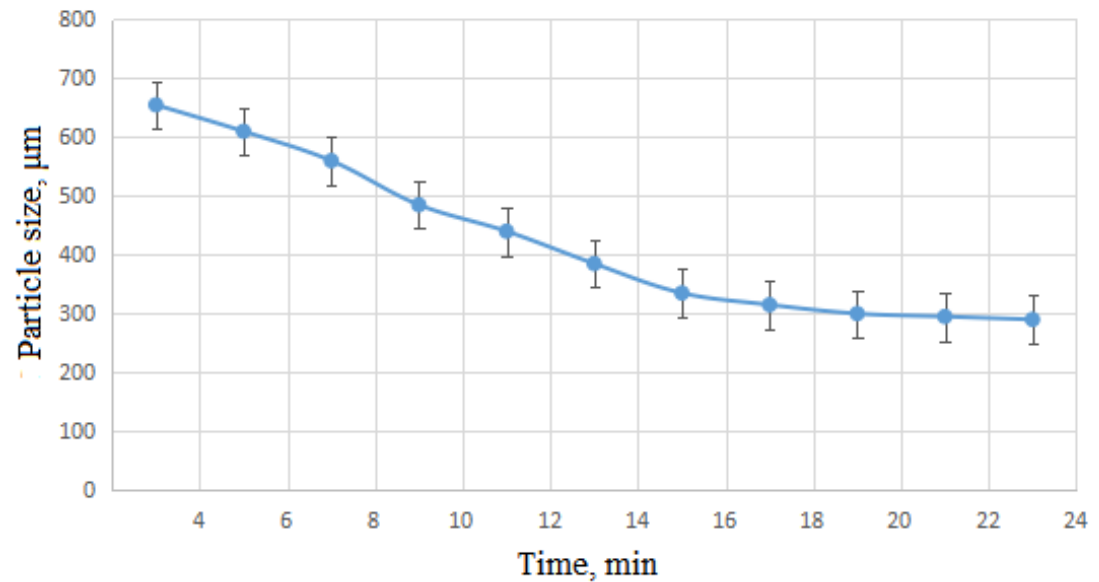

b)

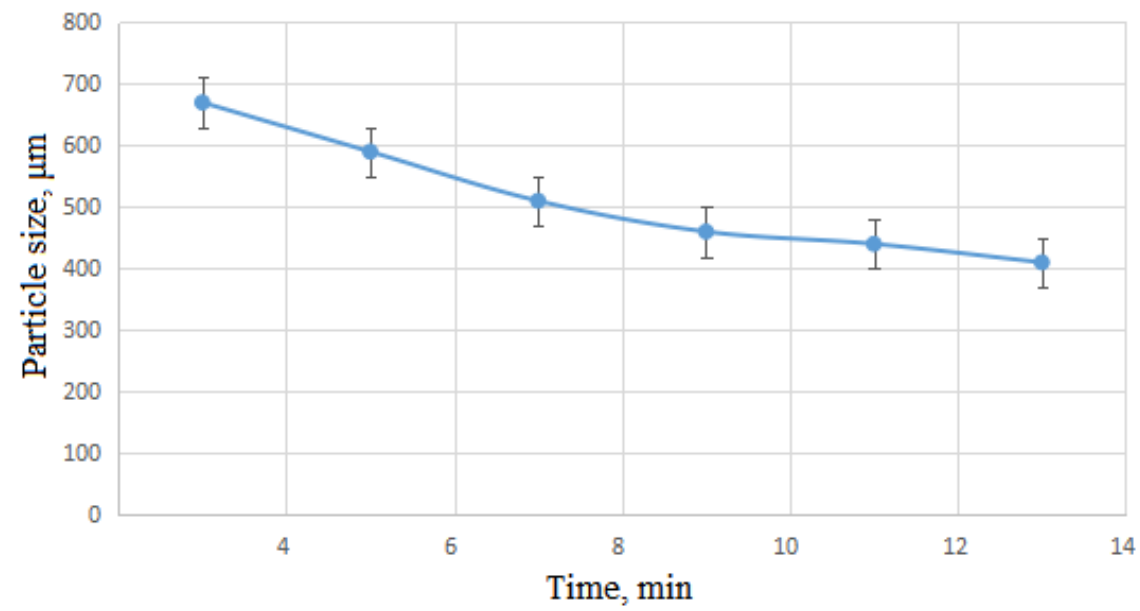

c)

Figure 12. Dependence of the average particle size of wheat in the liquid feed mixture on the processing time at different grain concentrations:

a) $10 \%$; b) $20 \%$; c) $30 \%$ 
As follows from the obtained dependences, the processing time of the grain mixture at a grain concentration of $10 \%$ is about 25 minutes or 30 cycles of processing the mixture in the working chamber of RPA. Increasing the grain concentration in the mixture to $20 \%$ reduces the processing time, which is 20 minutes or 25 processing cycles in the working chamber. At a wheat grain concentration of $30 \%$ in the watergrain mixture, the number of processing cycles decreases.

Analysis of the obtained dependences for different types of grains shows that the required processing time of the liquid grain mixture is $20-25$ minutes or $25-30$ processing cycles. With this duration of preparation of liquid feed, the average particle size of the grain will be close to $300 \mu \mathrm{m}$, which is the most optimal for its assimilation by domestic animals. It is necessary that the feed grain mixture has undergone 25-30 cycles of processing in the working chamber of RPA.

\subsubsection{Investigation of RPA energy characteristics during water-grain mixture processing.}

An important parameter of RPA for the preparation of liquid grain feed is to determine the required energy costs for the preparation of a unit of feed production. To determine this parameter, a wattmeter CTK3 - 10A 1H6PB was used, which was used to measure the power consumption of RPA motor at different points in time for different grain concentrations in the feed mixture. The research results are shown in Fig. 13.

In Fig. 13a shows the values of power consumption at different points in time and at a wheat concentration $10 \%$ in the feed mixture. Analysis of the obtained data shows that at the initial time the power consumption is maximum and gradually decreases during the processing of the water-grain environment. Increasing the concentration of wheat in the feed mixture leads to an increase in consumption capacity with less time spent on processing the water-grain environment (Fig. 13c).

It is characteristic that at the last stages of preparation of forage mix at high concentration of grain there is an increase in the consumed power of the electric motor. 
This fact is due to the increase in the viscosity of the feed mixture when it is heated in the last stages of processing.

It should be noted that the highest engine power consumption, as for the watergrain mixture using wheat, is observed in the initial stages of processing, when most of the energy is spent on grinding grain. With an increase in the concentration of grain fraction in 3 times (Fig. 13) energy costs also increase. However, in the final stages of processing, power consumption, as shown in experimental studies, is almost independent of concentration of corn grain in liquid feed.

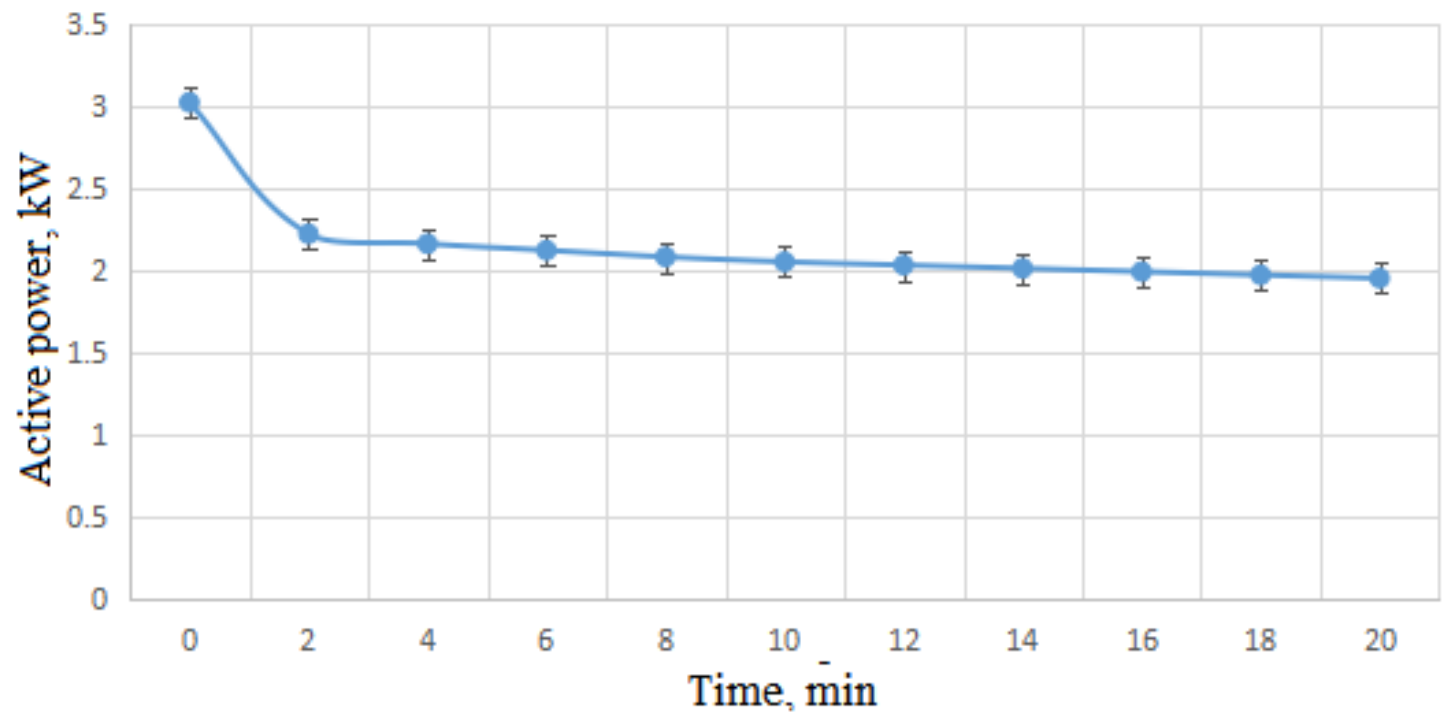

a)

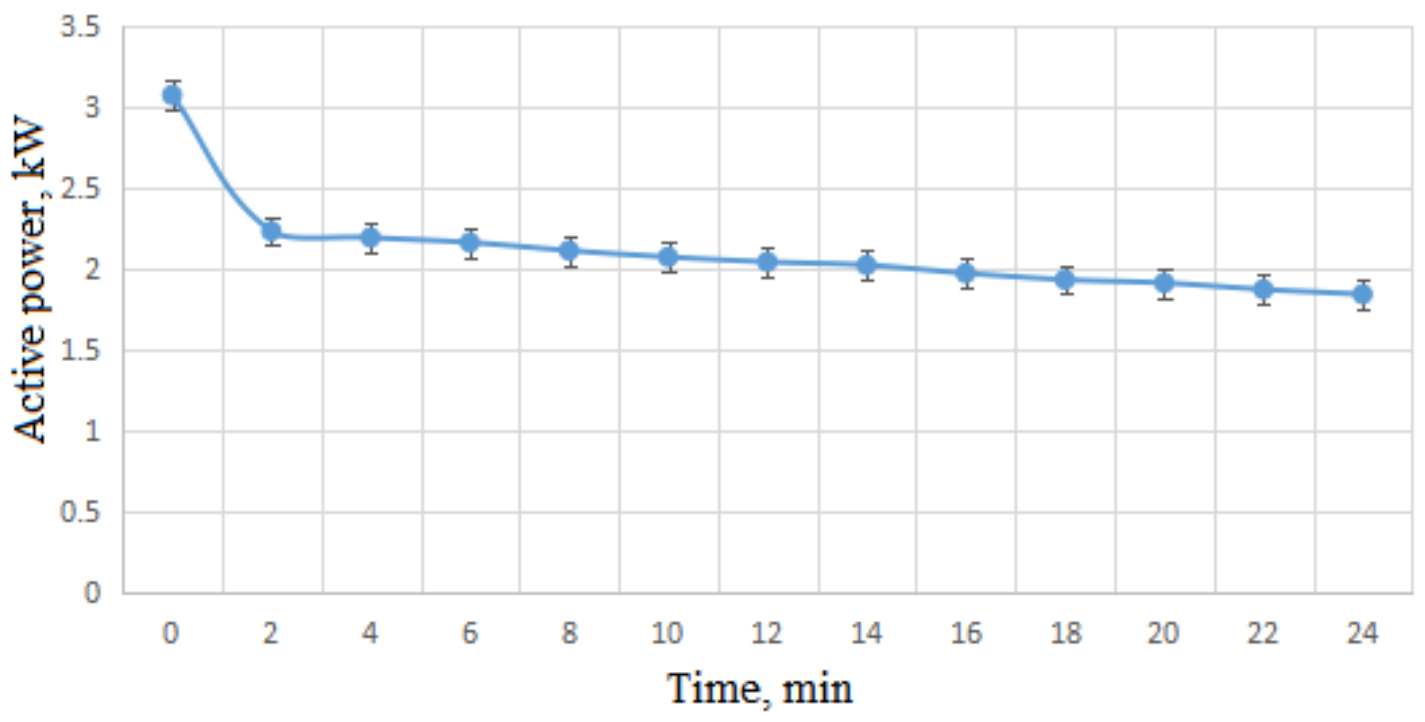

b) 


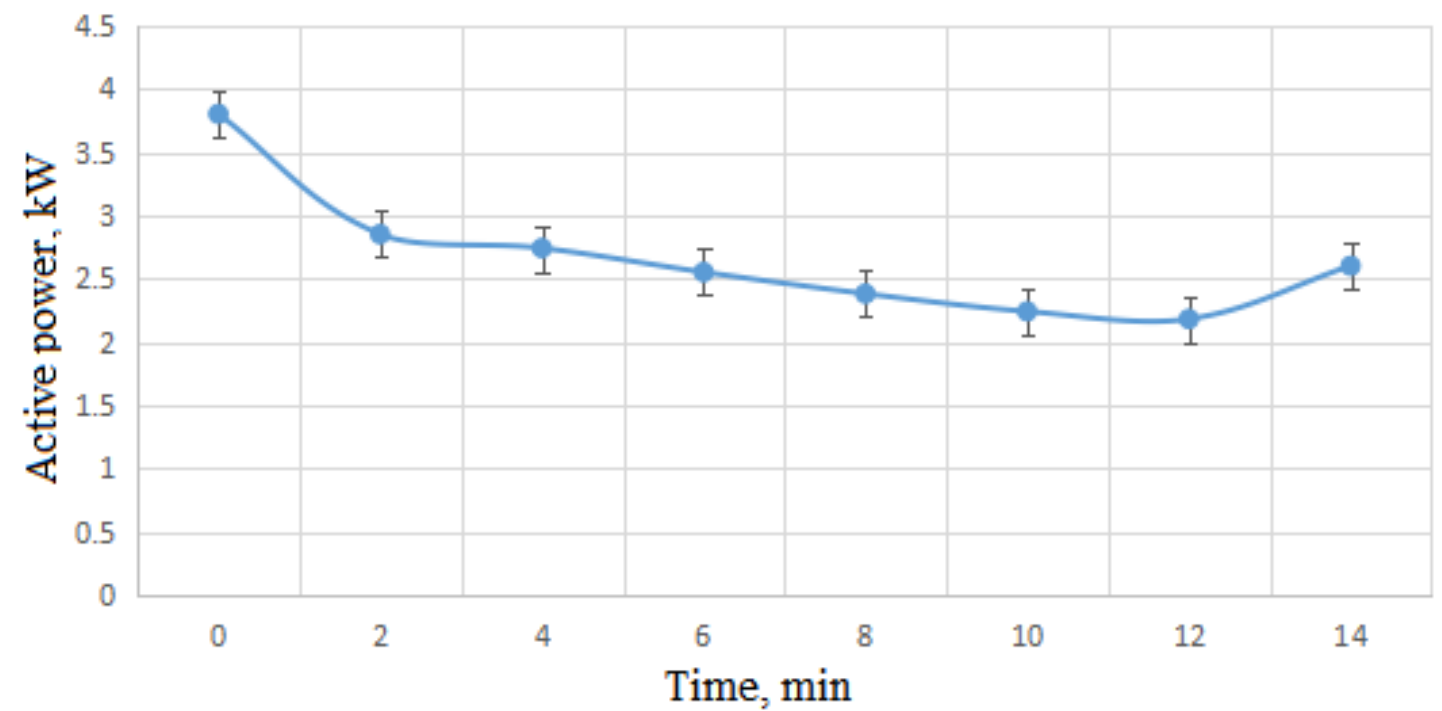

c)

Figure 13. Change in power consumption when processing the feed mixture at different concentrations of wheat in the mixture:

a) $10 \%$; b) $20 \%$; c) $30 \%$

\subsubsection{Investigation of thermal effects in RPA during treatment of water-grain medium.}

Temperature characteristics of liquid grain feeds play an important role in their preparation, as additional energy consumption is required to heat the feed. In this regard, the temperature of the feed mixture was measured depending on the processing time using wheat, corn and barley. The results of the study are shown in Fig. 14.

In Fig. 14 presents the temperature dependences of the wheat mixture at a grain concentration in the feed, respectively $10 \%, 20 \%$ and $30 \%$. The obtained dependences indicate an increase in the temperature of liquid grain feed with increasing time during its processing. These processes are due to the scattering of the kinetic energy of rotation of the rotor and its conversion into thermal energy. As follows from the obtained graphical dependences, with a higher concentration of grain in the water-grain mixture, the temperature rise will be higher, which is caused by higher energy consumption for its preparation. 


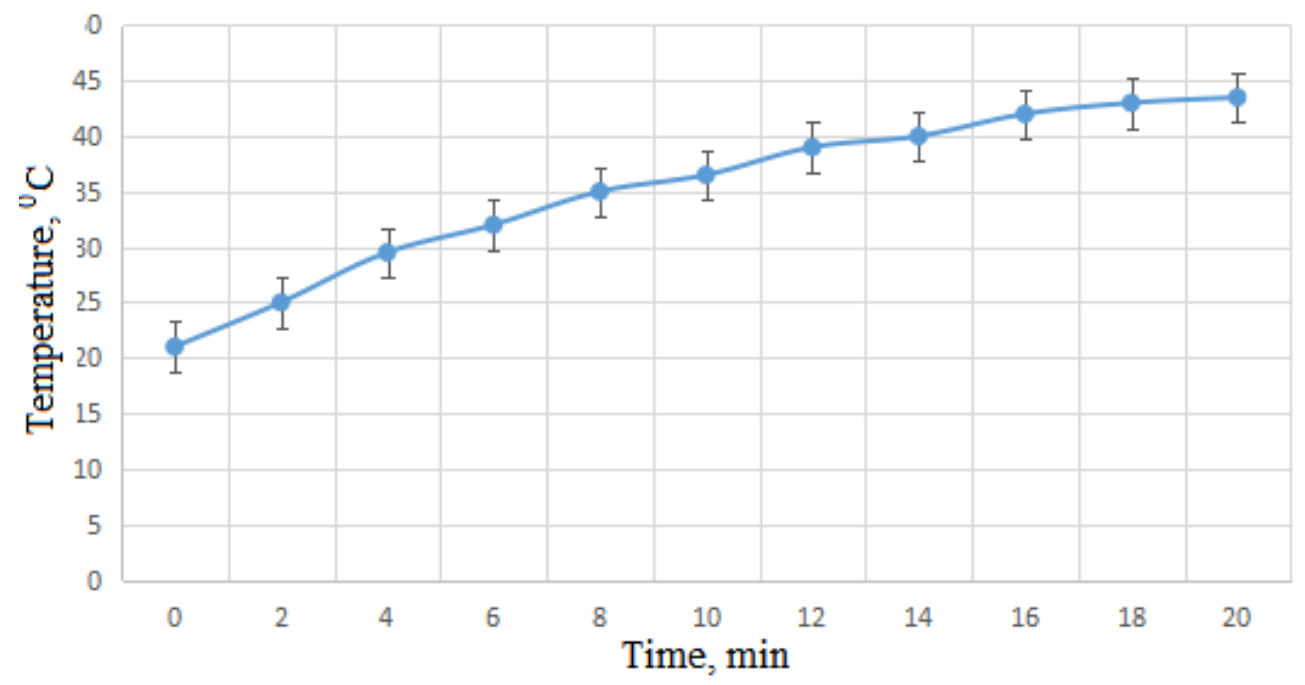

a)

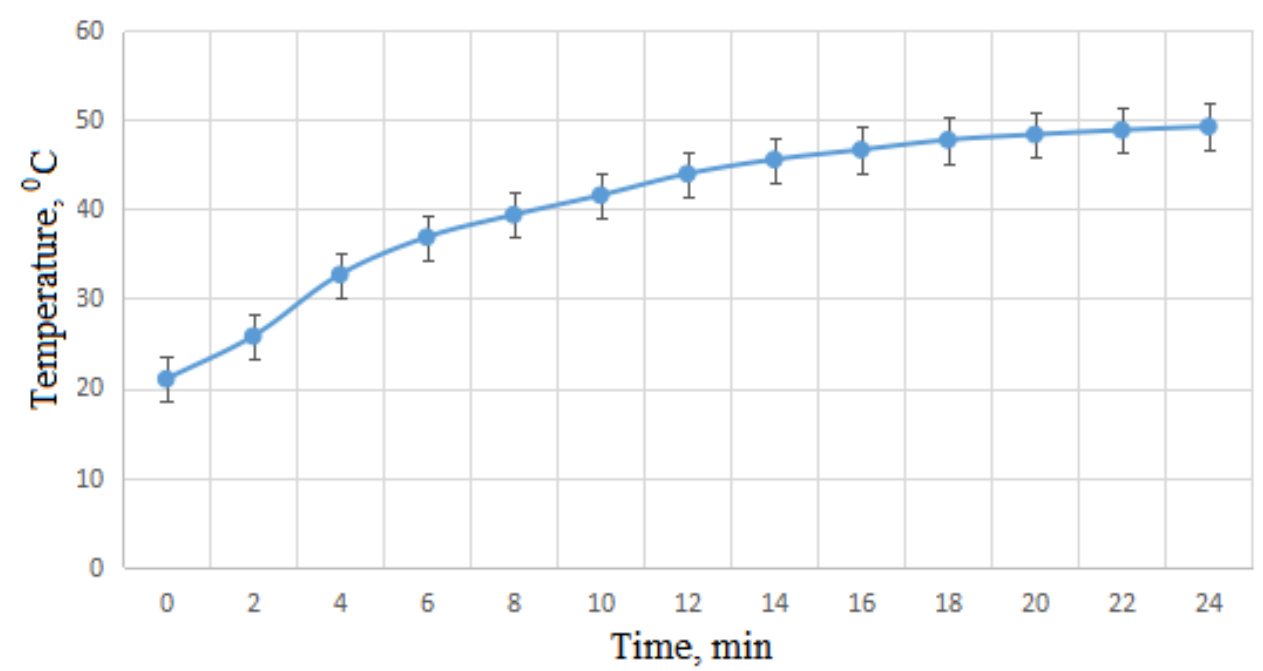

b)

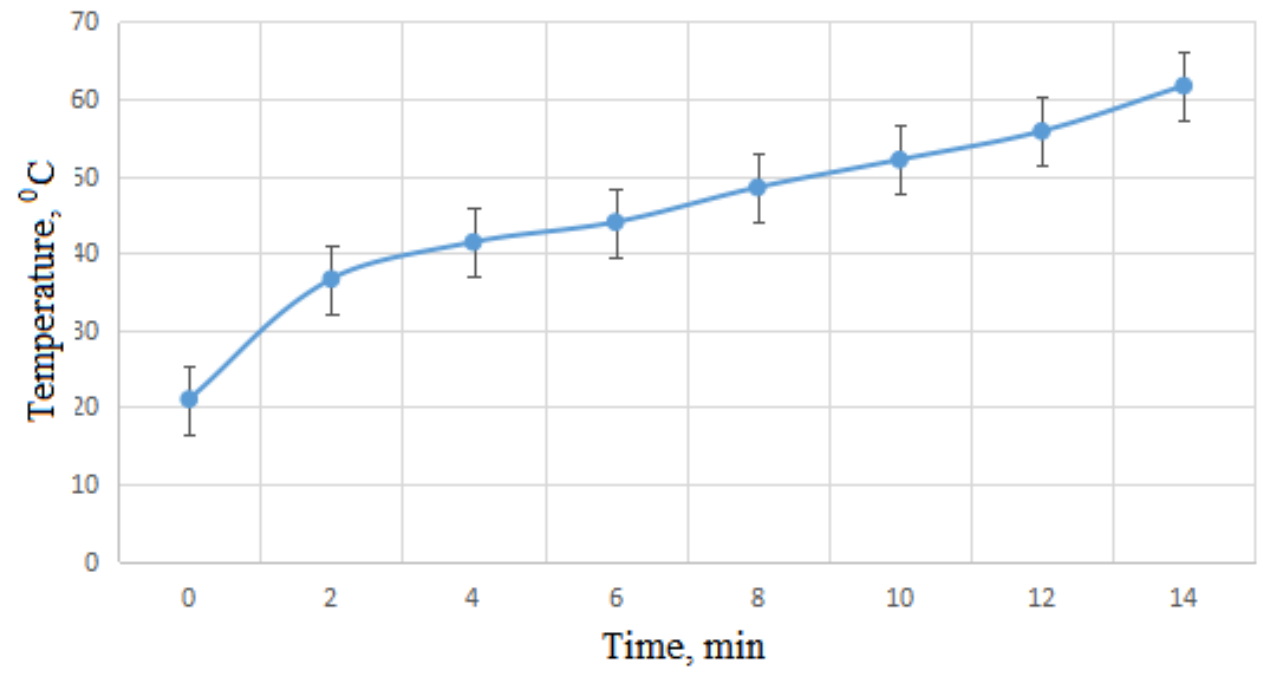

c)

Figure 14. The dependence of the temperature of the liquid grain mixture at different concentrations of wheat: a) 10\%; b) 20\%; c) $30 \%$ 
Thus, we can conclude that the use of discrete-pulse energy input into the starchcontaining medium, which is a water-grain mixture, is accompanied by the dissipation of the kinetic energy of rotation of the rotor into thermal energy. These phenomena are due turbulence, cavitation and other processes in the fluid flow that occur in the working chamber of RPA during the preparation of liquid grain feed.

For preparation of liquid highly dispersed forages by DPEI method it is necessary to mix 1 part of grain with 2 parts of water, ie on $70 \mathrm{~kg}$ of water $30 \mathrm{~kg}$ of grain are necessary. For 15 minutes of operation of the device the output of finished goods makes $100 \mathrm{~kg}$, and for 1 hour $-450-500 \mathrm{~kg}$.

From Fig. 13c shows that for the preparation of $100 \mathrm{~kg}$ of feed for 13 minutes requires $0.42 \mathrm{~kW} / \mathrm{h}$ of electricity. The consumption of electricity per 1 ton of finished product is $4.2 \mathrm{~kW} / \mathrm{h}$. The calculation of the economic effect from the introduction of one RPA with a capacity of $450-500 \mathrm{~kg} / \mathrm{h}$ for the finished product is given below.

Table 3

Economic effect from the preparation of $300 \mathrm{~kg}$ of liquid feed with the use of RPA

\begin{tabular}{|c|c|c|c|c|c|c|}
\hline spending & \multicolumn{3}{|c|}{ existing technology } & \multicolumn{3}{c|}{ DPEI method } \\
\hline \multirow{2}{*}{$\begin{array}{c}\text { energy } \\
\text { consumpti } \\
\text { on }\end{array}$} & $\mathrm{kW} / \mathrm{h}$ & $\begin{array}{c}\text { price for 1 } \\
\mathrm{kW} / \mathrm{h}, \\
\mathrm{UAH}\end{array}$ & $\begin{array}{c}\text { cost, } \\
\mathrm{UAH}\end{array}$ & $\mathrm{kW} / \mathrm{h}$ & $\begin{array}{c}\text { price for 1 } \\
\mathrm{kW} / \mathrm{h}, \\
\mathrm{UAH}\end{array}$ & $\begin{array}{c}\text { cost, } \\
\mathrm{UAH}\end{array}$ \\
\cline { 2 - 7 } & 9.10 & 1.68 & 15.29 & 4.20 & 1.68 & 7.06 \\
\hline $\begin{array}{c}\text { raw } \\
\text { materials } \\
\text { costs }\end{array}$ & $\begin{array}{c}\text { number, } \\
\mathrm{kg}\end{array}$ & $\begin{array}{c}\text { price for } 1 \mathrm{~kg}, \\
\mathrm{UAH}\end{array}$ & $\begin{array}{c}\text { cost, } \\
\mathrm{UAH}\end{array}$ & $\begin{array}{c}\text { number, } \\
\mathrm{kg}\end{array}$ & $\begin{array}{c}\text { price for 1 } \\
\mathrm{kg}, \mathrm{UAH}\end{array}$ & $\begin{array}{c}\text { cost, } \\
\mathrm{UAH}\end{array}$ \\
\cline { 2 - 7 } & 333.00 & 3.00 & 999.00 & 289.00 & 3.00 & 867.00 \\
\hline $\begin{array}{c}\text { general } \\
\text { expenses }\end{array}$ & \multicolumn{7}{|c|}{1014.29} & & 874.06 \\
\hline $\begin{array}{c}\text { economic } \\
\text { effect }\end{array}$ & \multicolumn{7}{|c|}{$1014.29-874.06=140.23 \mathrm{UAH}}$. \\
\hline
\end{tabular}


For comparison, the costs of the existing technology of feed preparation, which includes three separate stages: grinding (hammer crusher), mixing with water (mixing apparatus), heating (steam head). Table 3 shows the energy and resource costs of liquid feed preparation according to the existing technology and according to the technology using DPEI method.

Experimental studies have shown that liquid highly dispersed feed prepared by DPEI method has $15 \%$ greater nutritional and energy value and is more fully absorbed by animals than prepared by existing technology. Therefore, when calculating the economic effect, the costs by the amount of grain were reduced by the same amount.

To feed 100 pigs you need $1000 \mathrm{~kg}$ of feed per day, $360000 \mathrm{~kg}$ per year. The annual economic effect of feeding 100 pigs will be UAH 170000 .

\section{Conclusions}

1. New design of the rotor-pulsation apparatus has been developed, which uses a working chamber with rotor and stator having rectangular holes for the passage of the feed mixture.

2. As a result of numerical three-dimensional modeling of the dynamics of fluid motion in the rotary pulsation apparatus obtained pressure and temperature fields in the working area of the apparatus at different times, which allows you to choose the optimal geometric characteristics of rotary pulsation apparatus.

3. Developed and manufactured experimental setup and developed methods of experimental research, which made it possible to determine the kinematic and dynamic characteristics, as well as features of discrete-pulse energy input during dispersion of liquid grain mixture in a rotary pulsation apparatus. The chemical and biological properties of the obtained liquid grain mixtures have been studied.

4. It is experimentally established that the use of rotary pulsation apparatus in the technology of preparation of liquid grain feed for farm animals can reduce the energy consumption of the process by 2-3 times compared to traditional technology, as well as increase productivity by $10-15 \%$ on the finished product. 


\section{Acknowledgement}

Supported by Ministry of Education and Science of Ukraine project № 110/10-pr-2019 "Development of a new method of feed preparation using rotary pulsation devices to improve the quality of feed mixture" 